\title{
Enantioselective Rh-Catalyzed Hydrogenation of Enol Carbamates with Monodentate Phosphoramidites
}

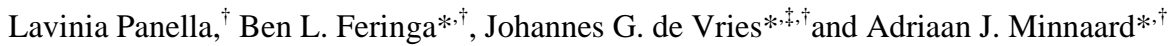

Department of Organic and Molecular Inorganic Chemistry, Stratingh Institute, University of Groningen, Nijenborgh 4, 9747 AG Groningen, The Netherlands.

DSM Research, Life Sciences-Advanced Synthesis, Catalysis and Development, P.O. Box 18, 6160 MD Geleen, The Netherlands.

b.l.feringa@rug.nl; hans-jg.vries-de@dsm.com; a.j.minnaard@rug.nl;

\section{SUPPORTING INFORMATION}

Table of contents:

${ }^{1}$ H-NMR

1-phenylvinyl acetate (1)

1-(4-chloro-phenyl)-vinyl acetate (2) S3

1-(4-nitro-phenyl)-vinyl acetate (3) S3

1-phenylethyl acetate (4) S4

1-(4-chloro-phenyl)-ethyl acetate (5) S4

1-(4-nitro-phenyl)-ethyl acetate (6) S5

1-phenylvinyl $N, N$-dimethylcarbamate (7) $\quad$ S5

1-phenylvinyl $N, N$-diethylcarbamate (8) $\quad$ S6

1-phenylvinyl $N, N$-diisopropylcarbamate (9) S6

1-phenylethyl $N, N$-dimethylcarbamate (10) $\quad \mathrm{S} 7$

$\begin{array}{ll}\text { 1-phenylethyl } N, N \text {-diethylcarbamate (11) } & \mathrm{S} 7\end{array}$

1-phenylethyl $N, N$-diisopropylcarbamate (12) S8

1-(4-nitro-phenyl)-vinyl $N, N$-diethylcarbamate (13) S8

1-butylvinyl $N, N$-diethylcarbamate (14) $\quad$ S9

1-benzylvinyl $N, N$-diethylcarbamate (15) $\quad$ S9

1-trimethylsilanylvinyl $N, N$-diethylcarbamate (16) $\quad$ S10

1-methylene-oct-2-enyl $N, N$-diethylcarbamate (17) $\quad \mathrm{S} 10$

1-methylene-3-phenyl-allyl N,N-diethylcarbamate (18) S11

vinyl $N, N$-diethylcarbamate (19) S11

1-(4-nitro-phenyl)-ethyl $N, N$-diethylcarbamate (20) $\quad \mathrm{S} 12$

1-methylpentyl $N, N$-diethylcarbamate (21) $\quad \mathrm{S} 12$

1-methyl-2-phenylethyl $N, N$-diethylcarbamate (22) S13

1-trimethylsilanylethyl $N, N$-diethylcarbamate (23) $\quad \mathrm{S} 13$

1-methyl-oct-2-enyl $N, N$-diethylcarbamate (24) $\quad \mathrm{S} 14$

1-methyl-3-phenyl-allyl $N, N$-diethylcarbamate (25) $\quad$ S14

${ }^{13}$ C-NMR $\quad$ Page

1-phenylvinyl $N, N$-dimethylcarbamate (7) $\quad$ S15

1-phenylvinyl $N, N$-diethylcarbamate (8) $\quad \mathrm{S} 15$ 
1-phenylvinyl $N, N$-diisopropylcarbamate (9)

1-phenylethyl $N, N$-dimethylcarbamate (10)

1-phenylethyl $N, N$-diethylcarbamate (11)

1-(4-nitro-phenyl)-vinyl $N, N$-diethylcarbamate (13)

1-butylvinyl $N, N$-diethylcarbamate (14)

S18

1-benzylvinyl $N, N$-diethylcarbamate (15)

1-trimethylsilanylvinyl $N, N$-diethylcarbamate (16)

S19

1-methylene-oct-2-enyl $N, N$-diethylcarbamate (17)

S19

1-methylene-3-phenyl-allyl $N, N$-diethylcarbamate (18)

S20

vinyl $N, N$-diethylcarbamate (19)

1-(4-nitro-phenyl)-ethyl $N, N$-diethylcarbamate (20)

1-methylpentyl $N, N$-diethylcarbamate (21)

1-methyl-2-phenylethyl $N, N$-diethylcarbamate (22)

1-trimethylsilanylethyl $N, N$-diethylcarbamate (23)

1-methyl-oct-2-enyl $N, N$-diethylcarbamate (24)

\section{1-phenylvinyl acetate (1)}

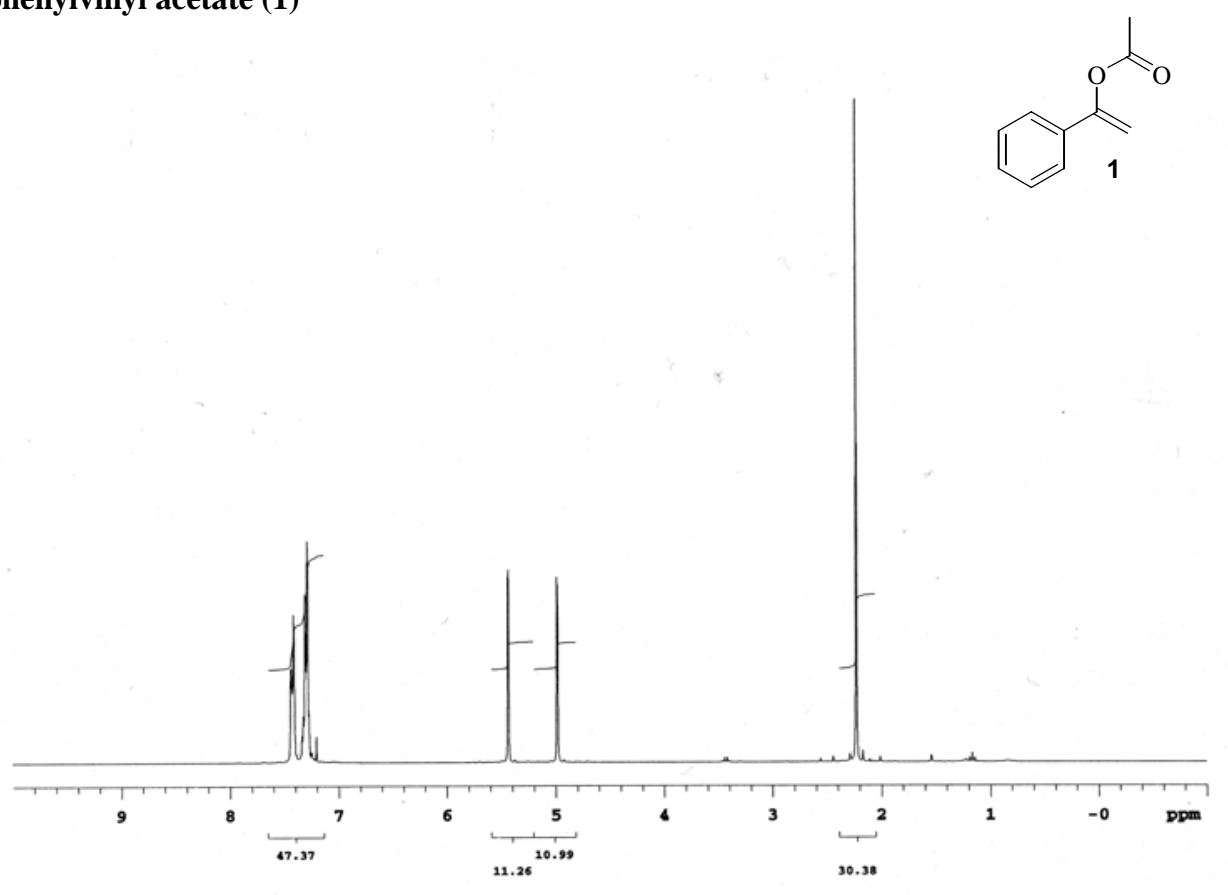


1-(4-chloro-phenyl)-vinyl acetate (2)

pules sequence: a2pol
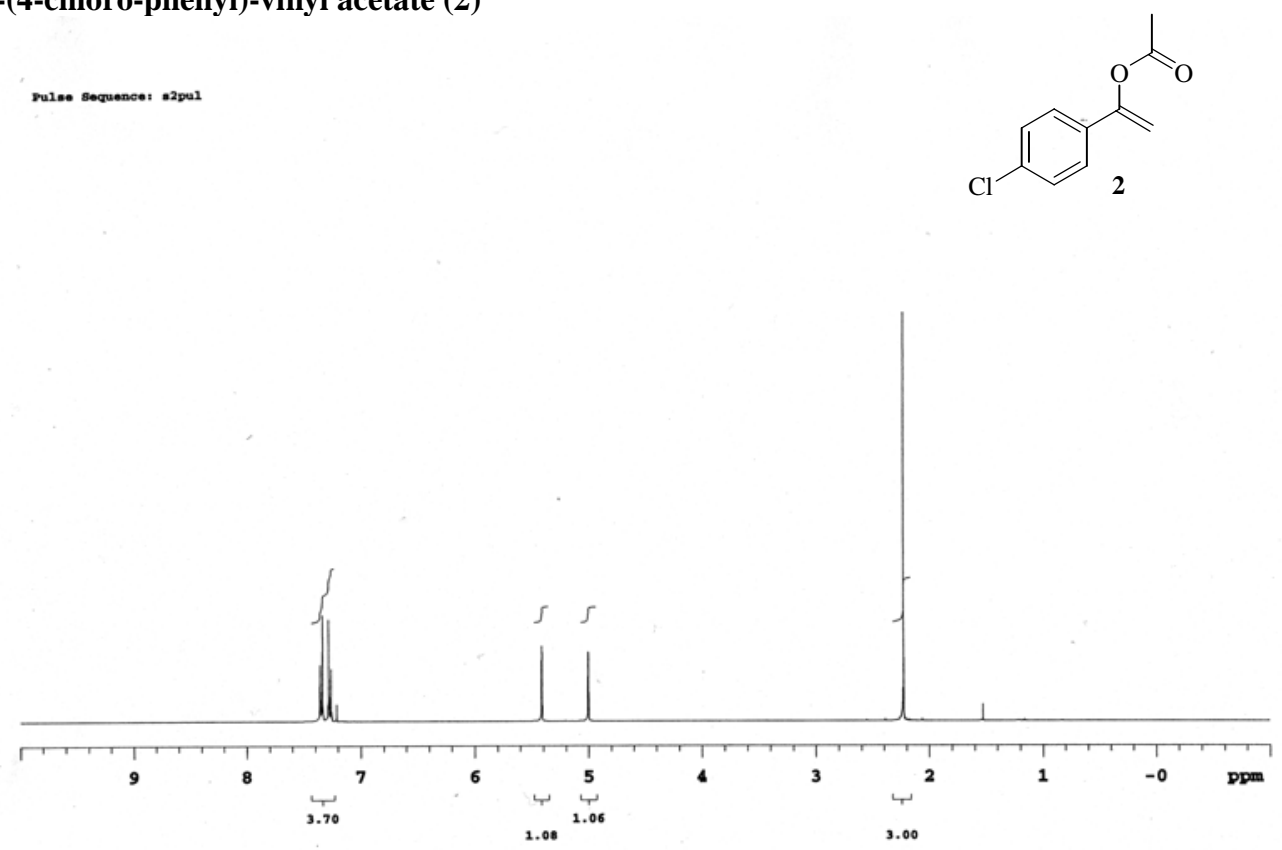

1-(4-nitro-phenyl)-vinyl acetate (3)
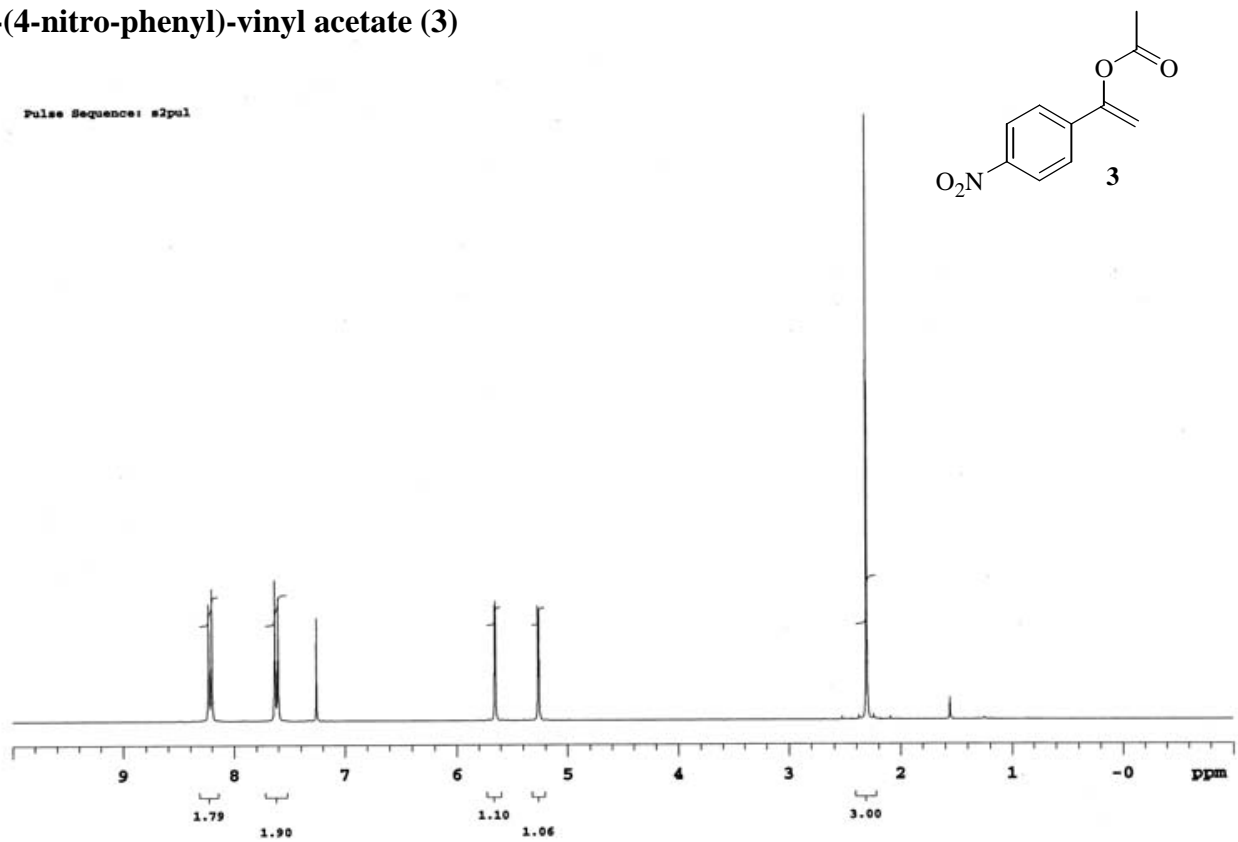
1-phenylethyl acetate (4)
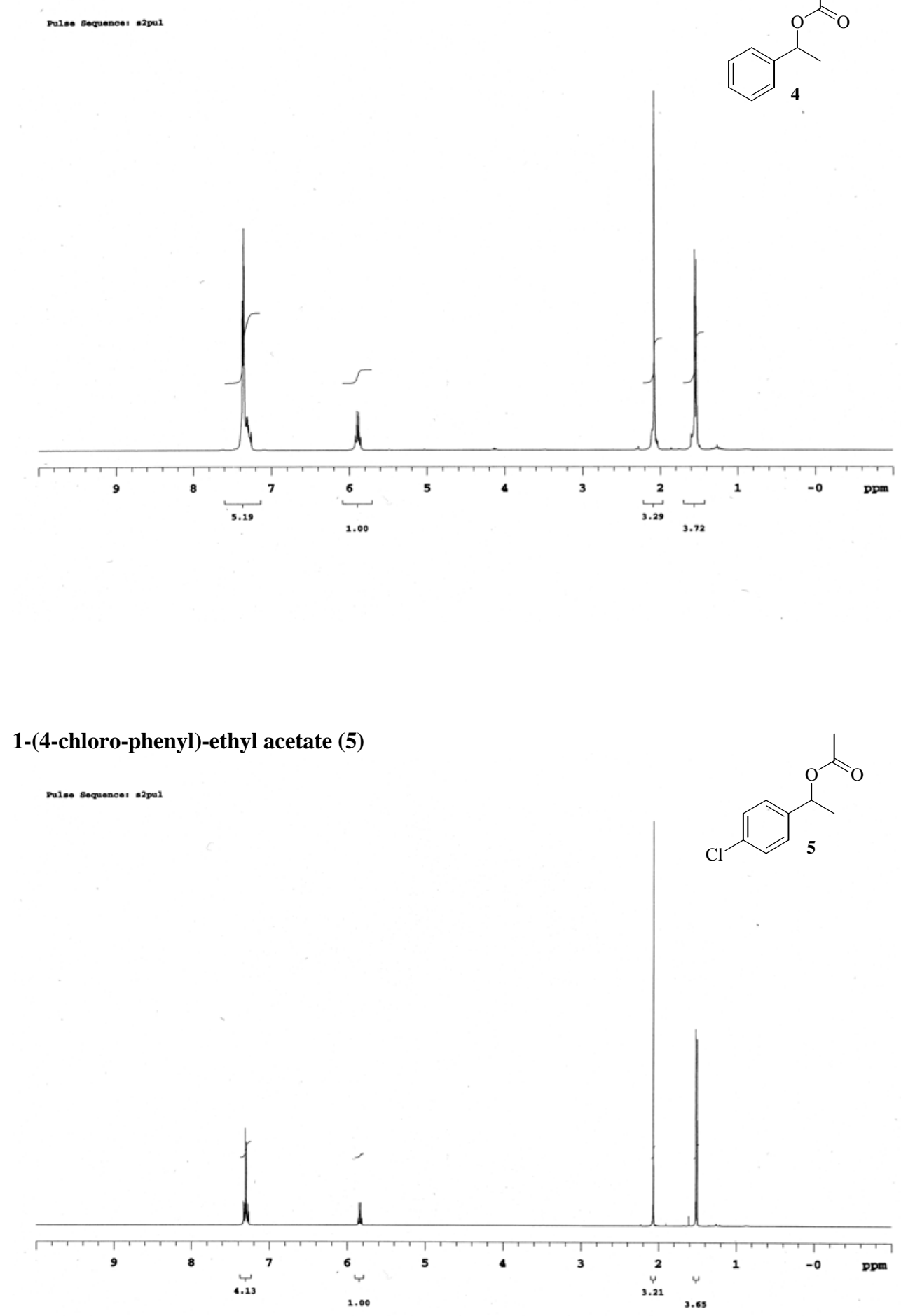
1-(4-nitro-phenyl)-ethyl acetate (6)

prales soquencoer s2pol
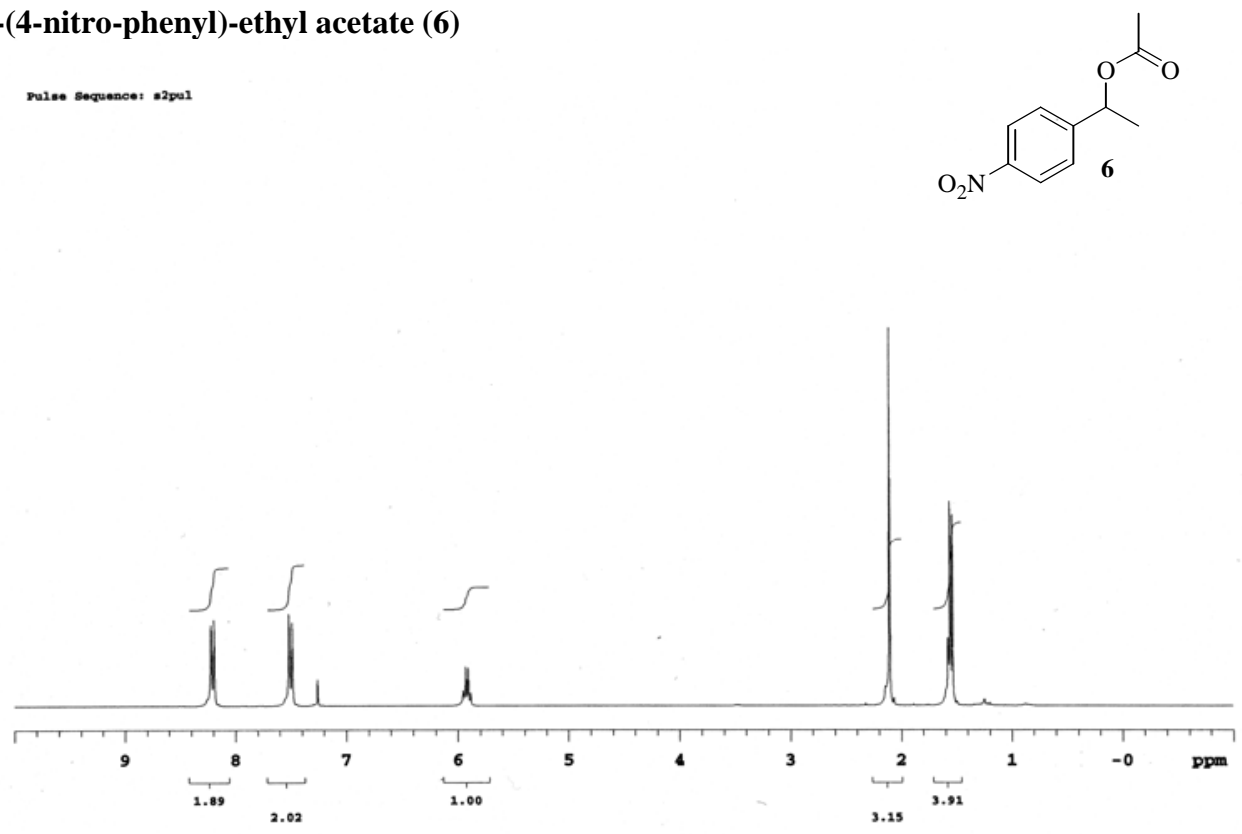

1-phenylvinyl dimethylcarbamate (7)

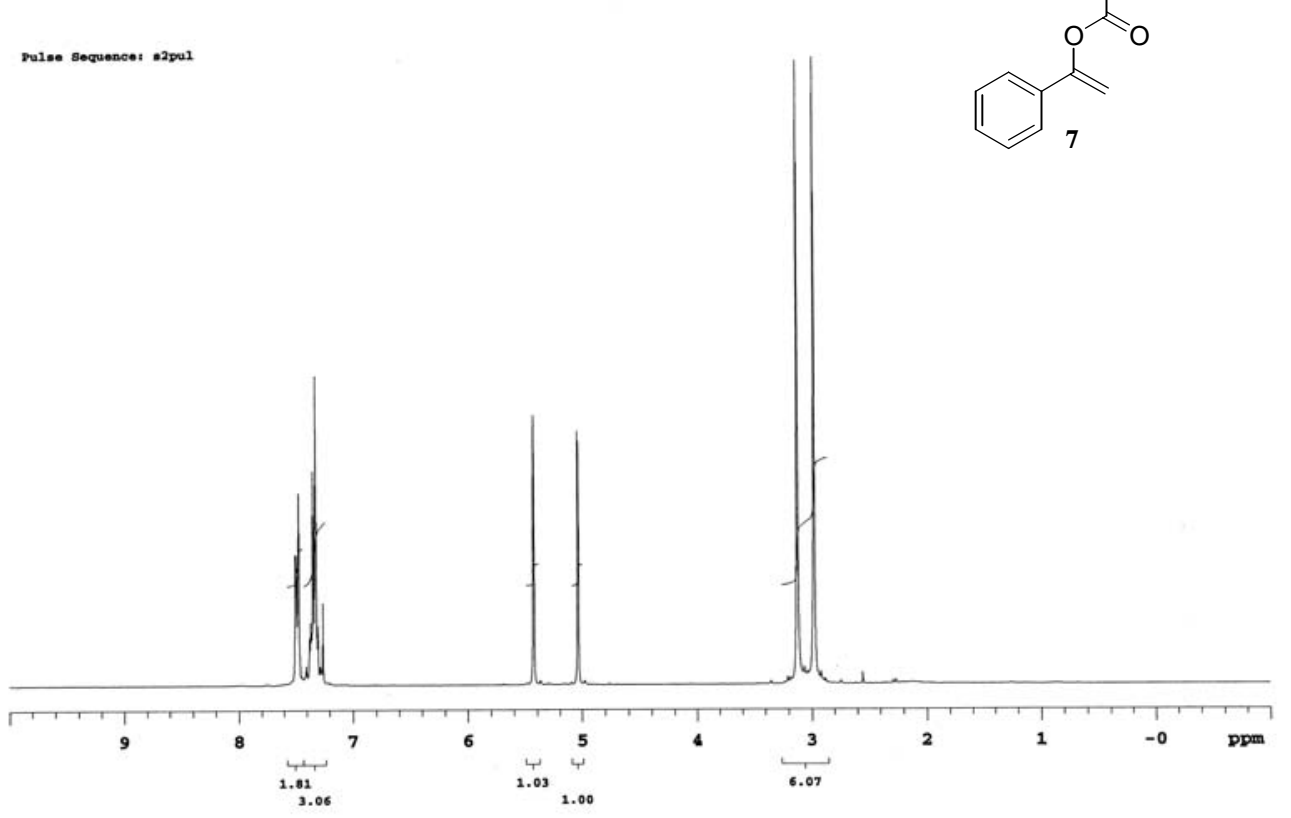


1-phenylvinyl diethylcarbamate (8)

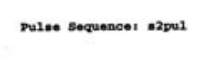

\section{(8)}

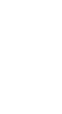


1-phenylethyl dimethylcarbamate (10)

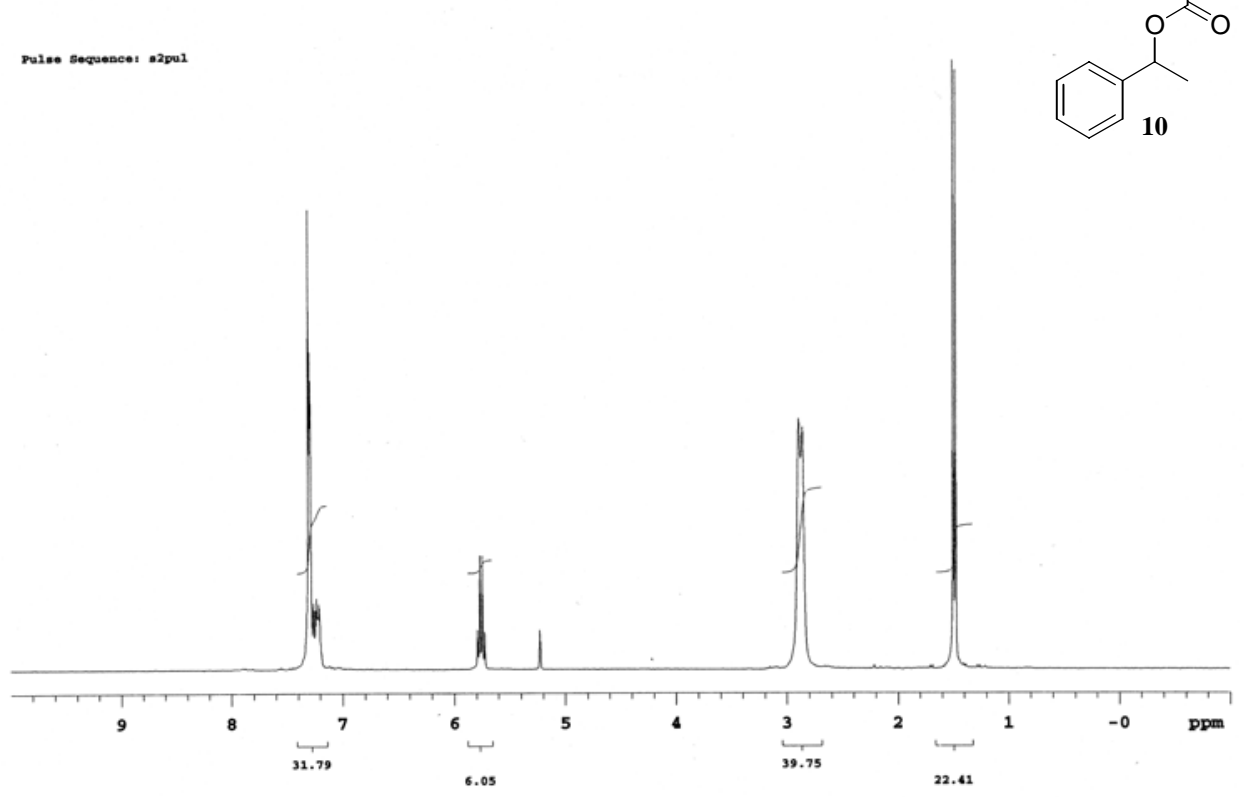

1-phenylethyl diethylcarbamate (11)

pules Bequescert s2pou
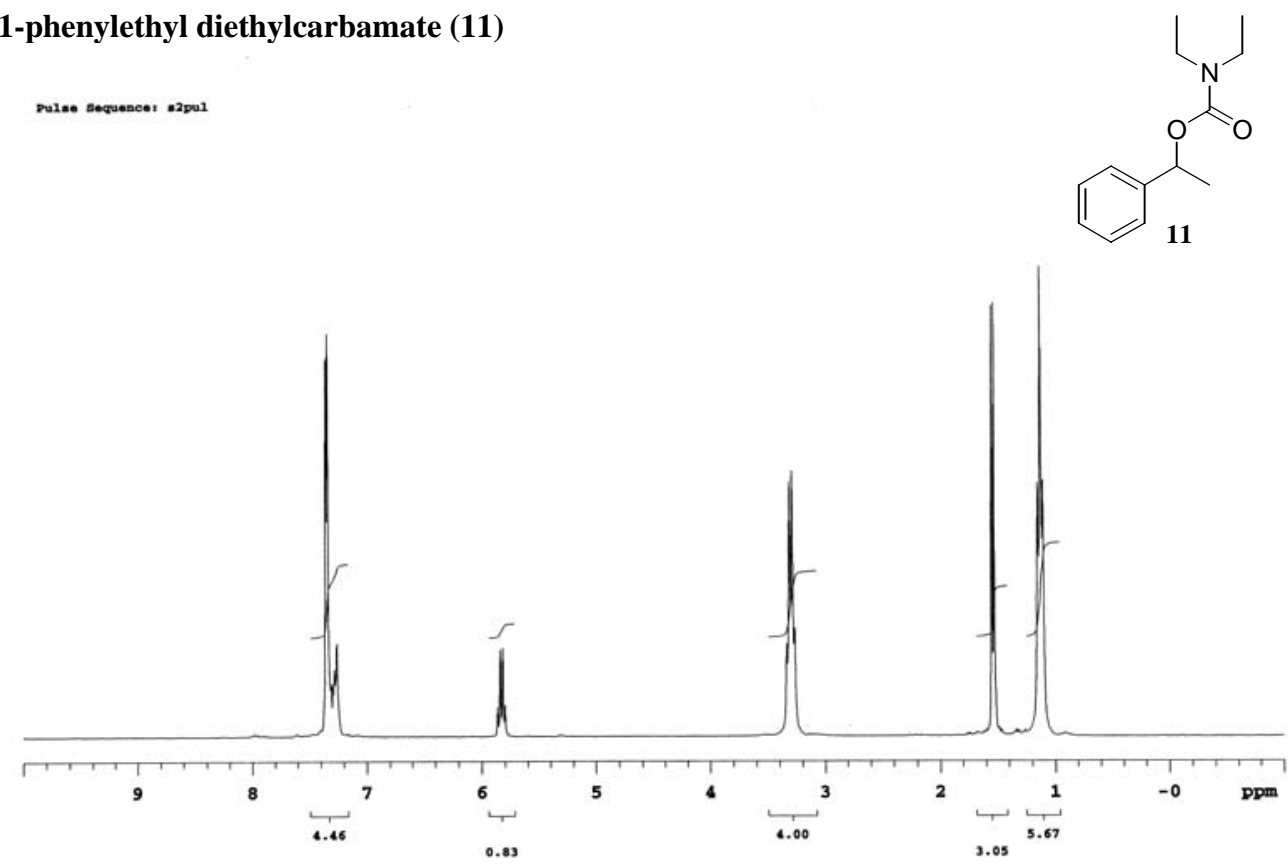
1-phenylethyl diisopropylcarbamate (12)
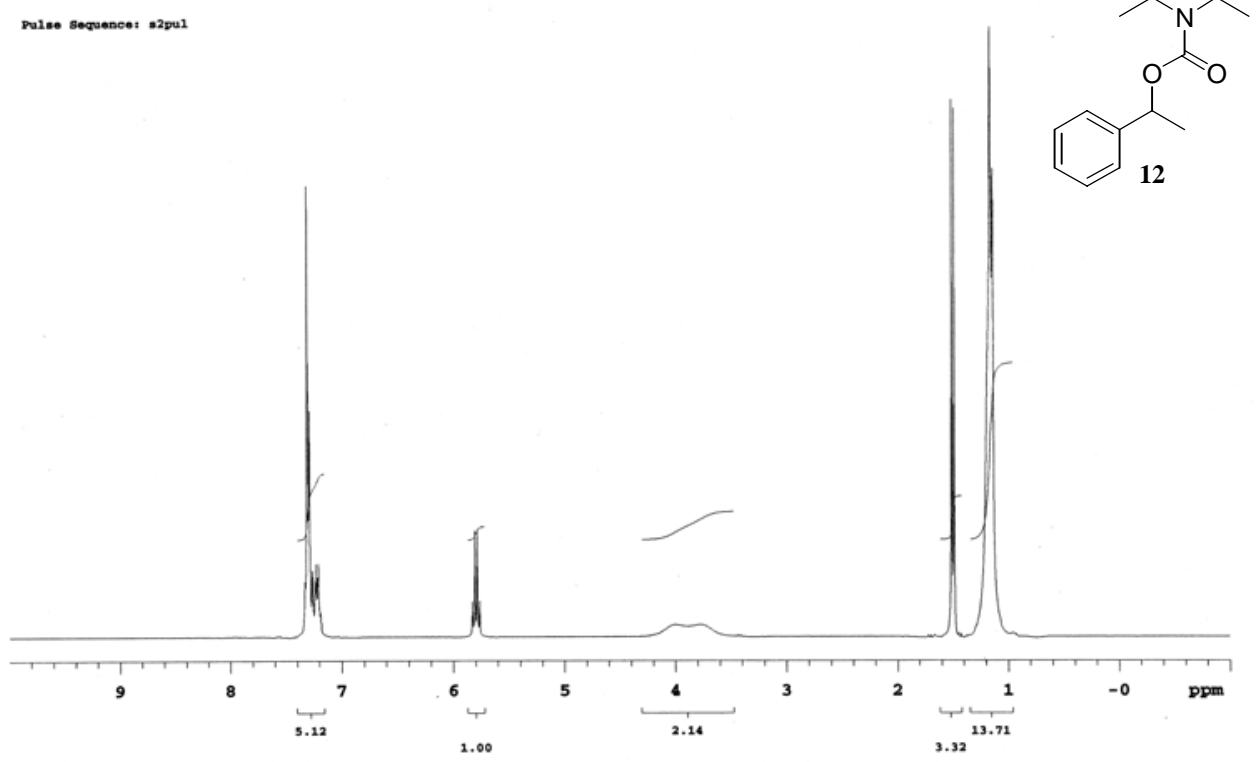

1-(4-nitro-phenyl)-vinyl diethylcarbamate (13)
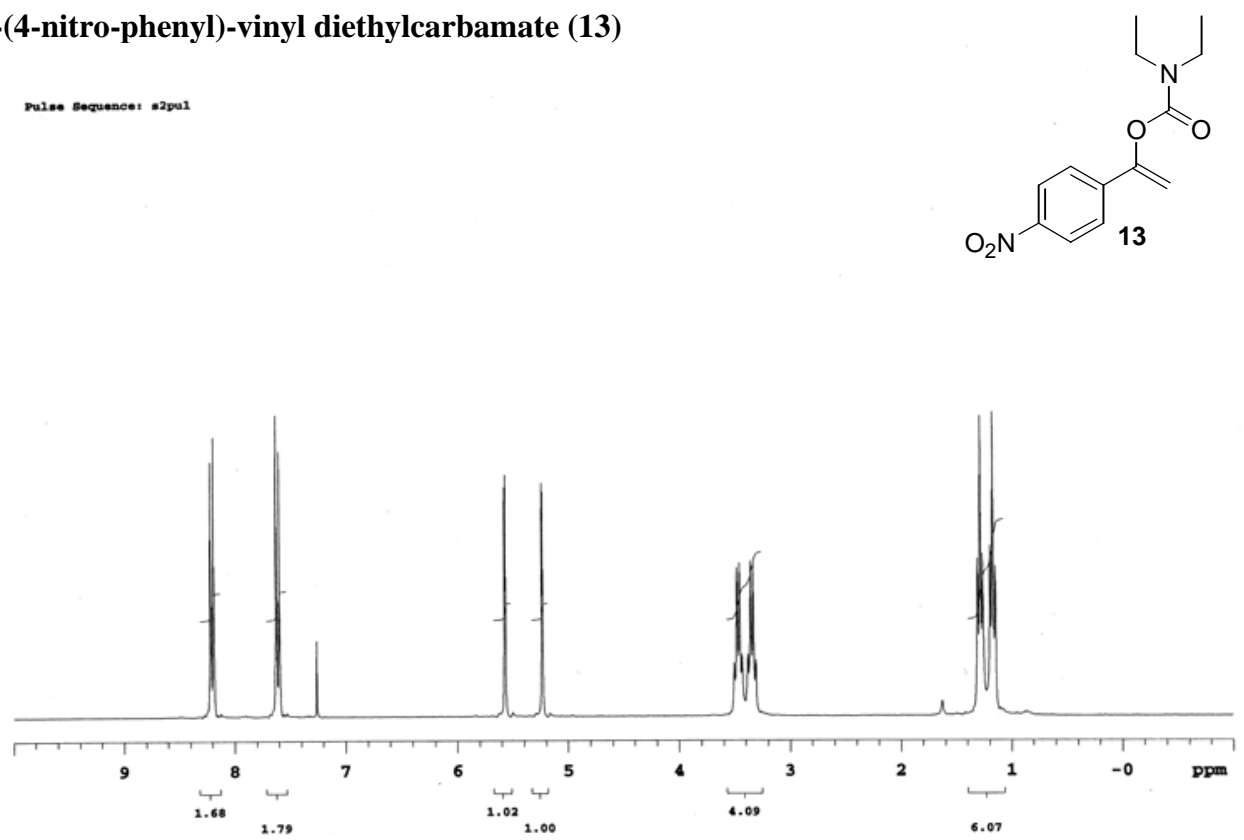
1-butylvinyl diethylcarbamate (14)

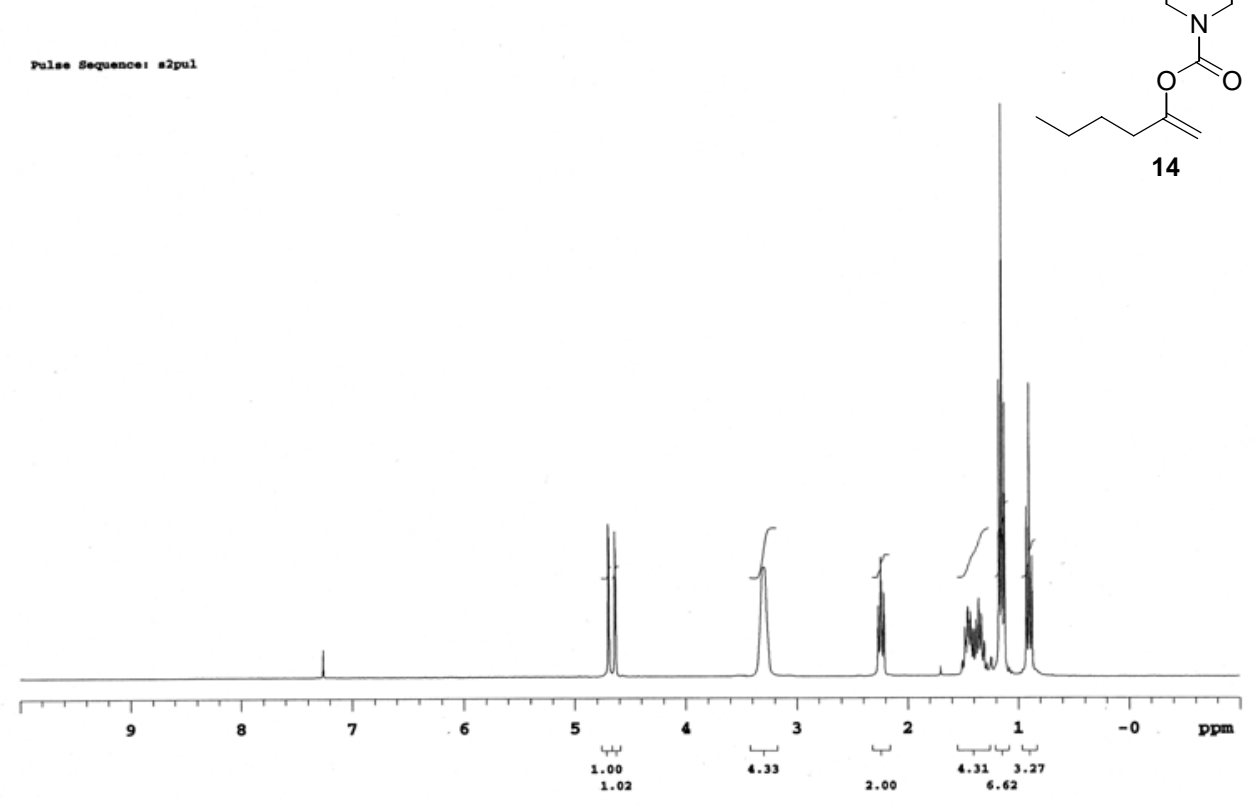

1-benzylvinyl diethylcarbamate (15)

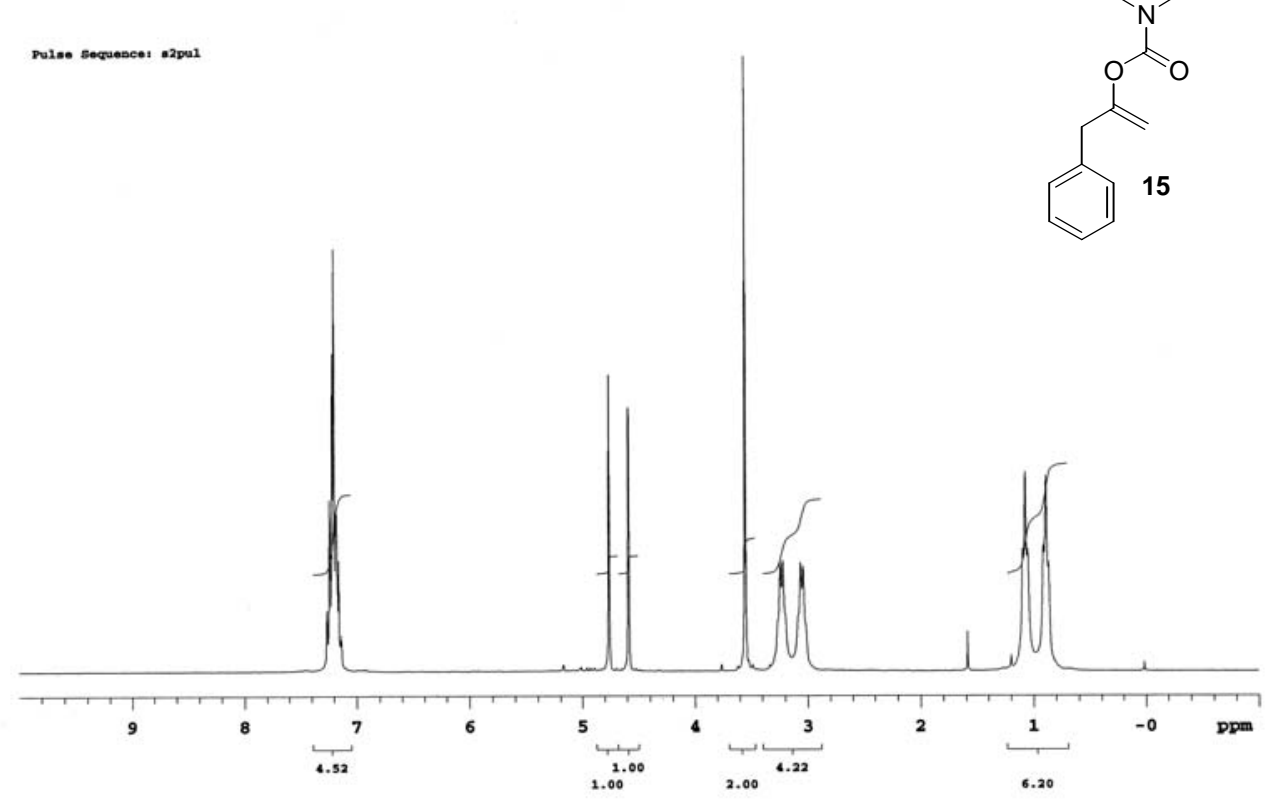


1-trimethylsilanylvinyl diethylcarbamate (16)
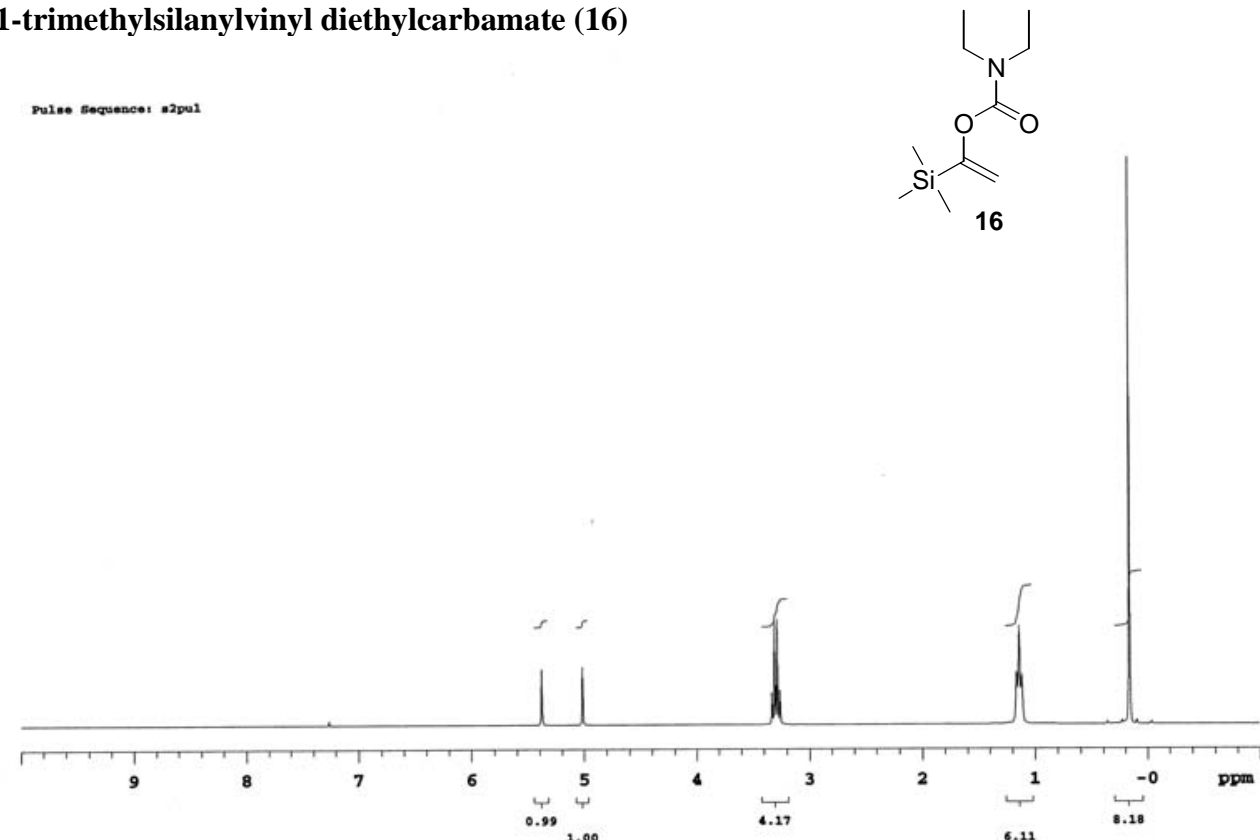

1-methylene-oct-2-enyl diethylcarbamate (17)

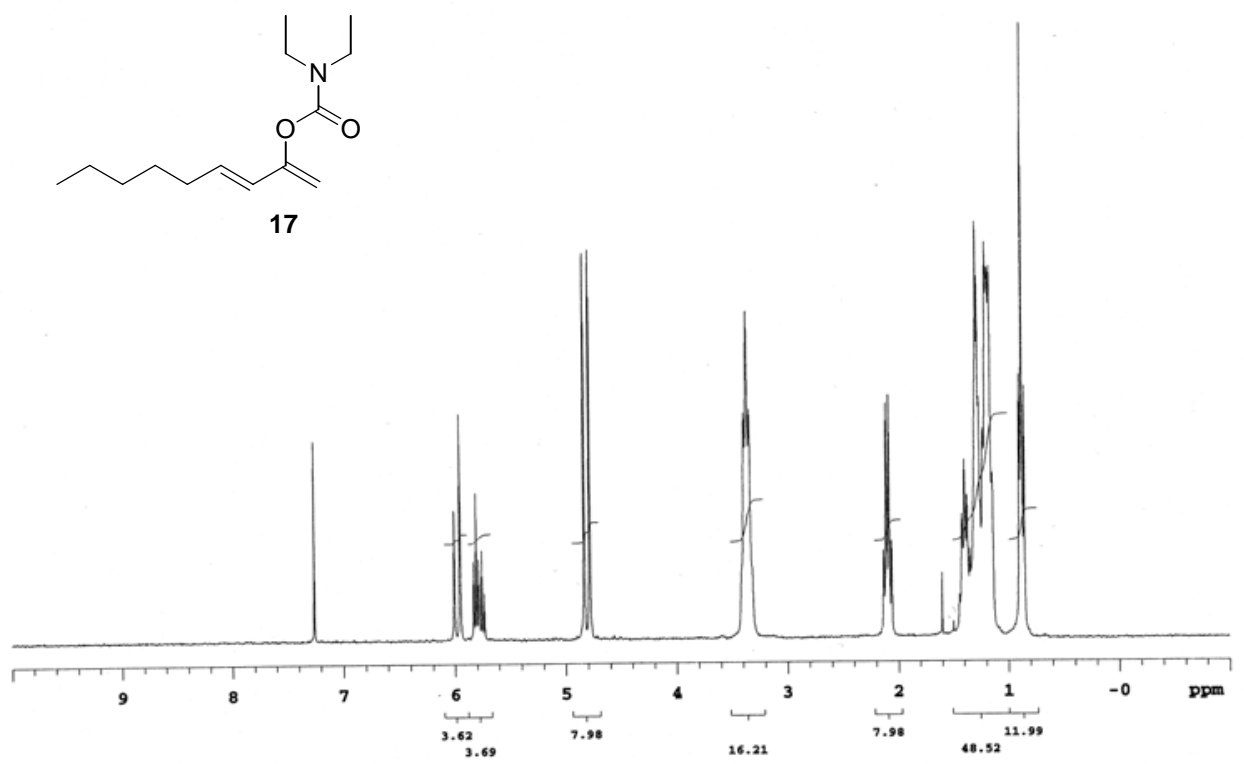


1-methylene-3-phenyl-allyl diethylcarbamate (18)

pules sequenceri sapul
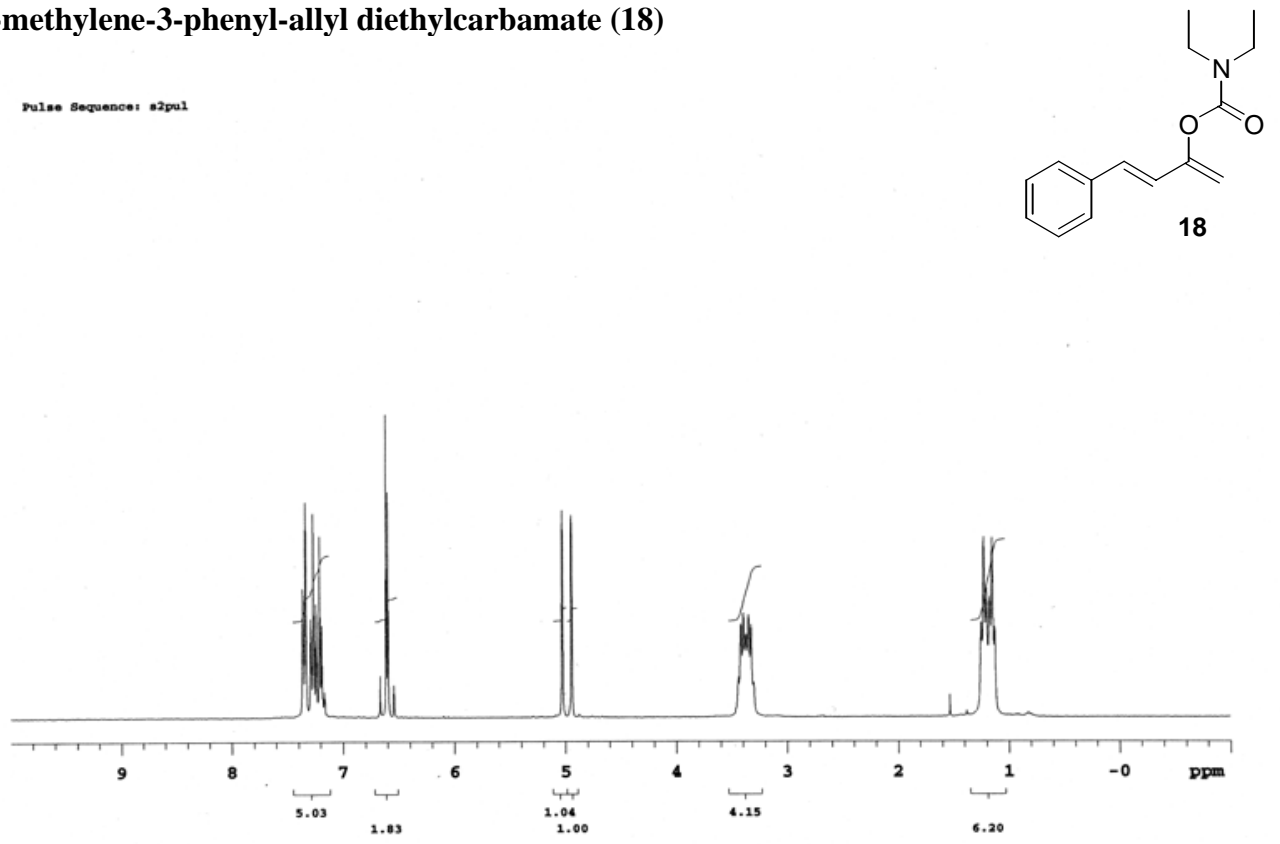

vinyl diethylcarbamate (19)
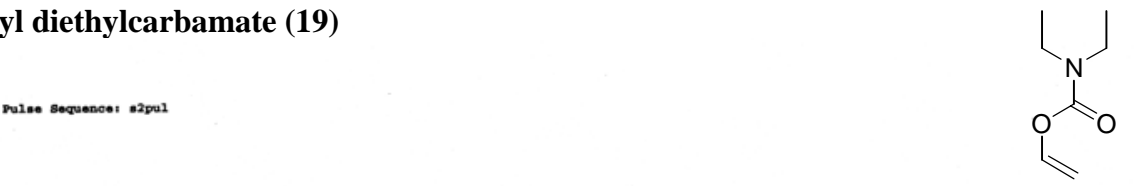

19

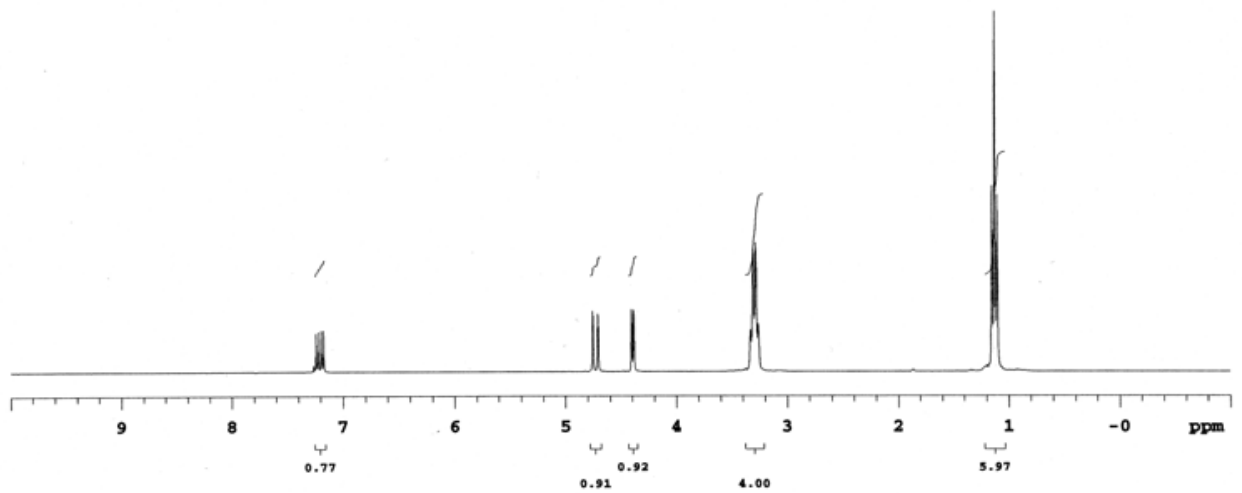


1-(4-nitro-phenyl)-ethyl diethylcarbamate (20)

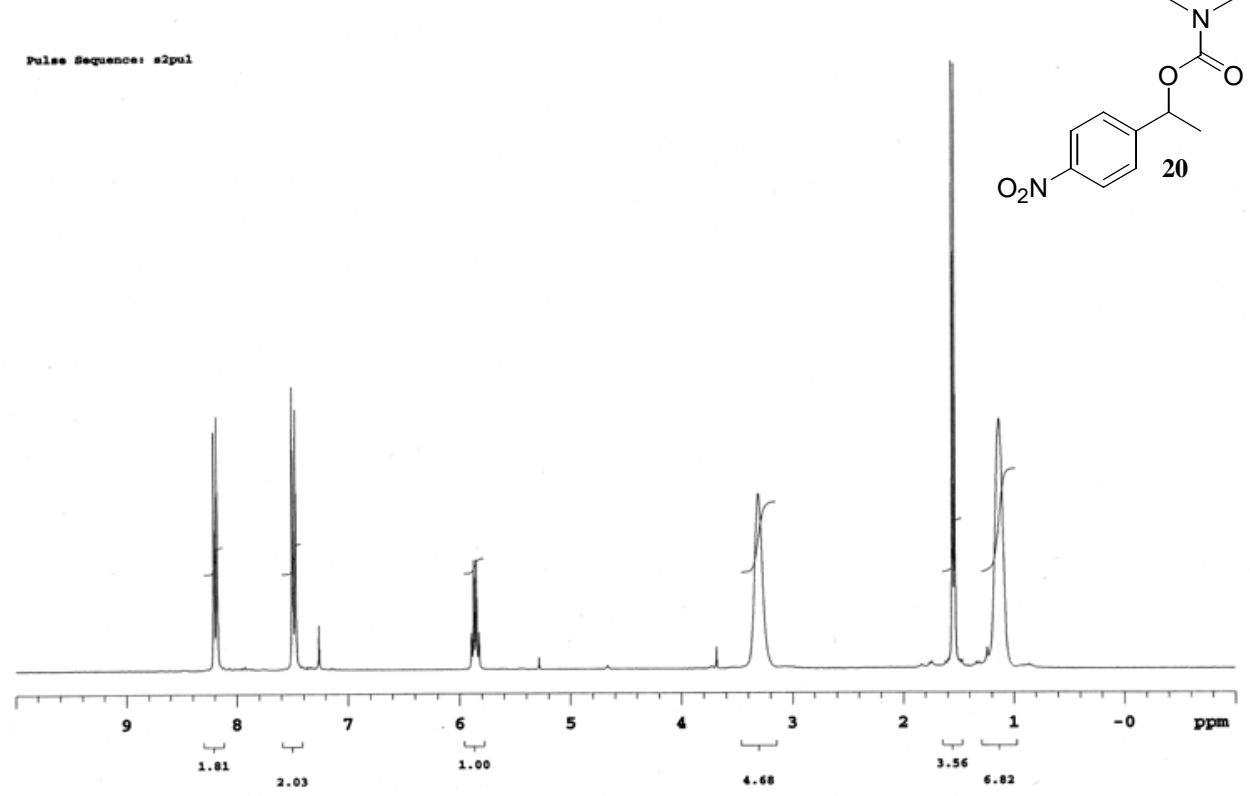

1-methylpentyl diethylcarbamate (21)

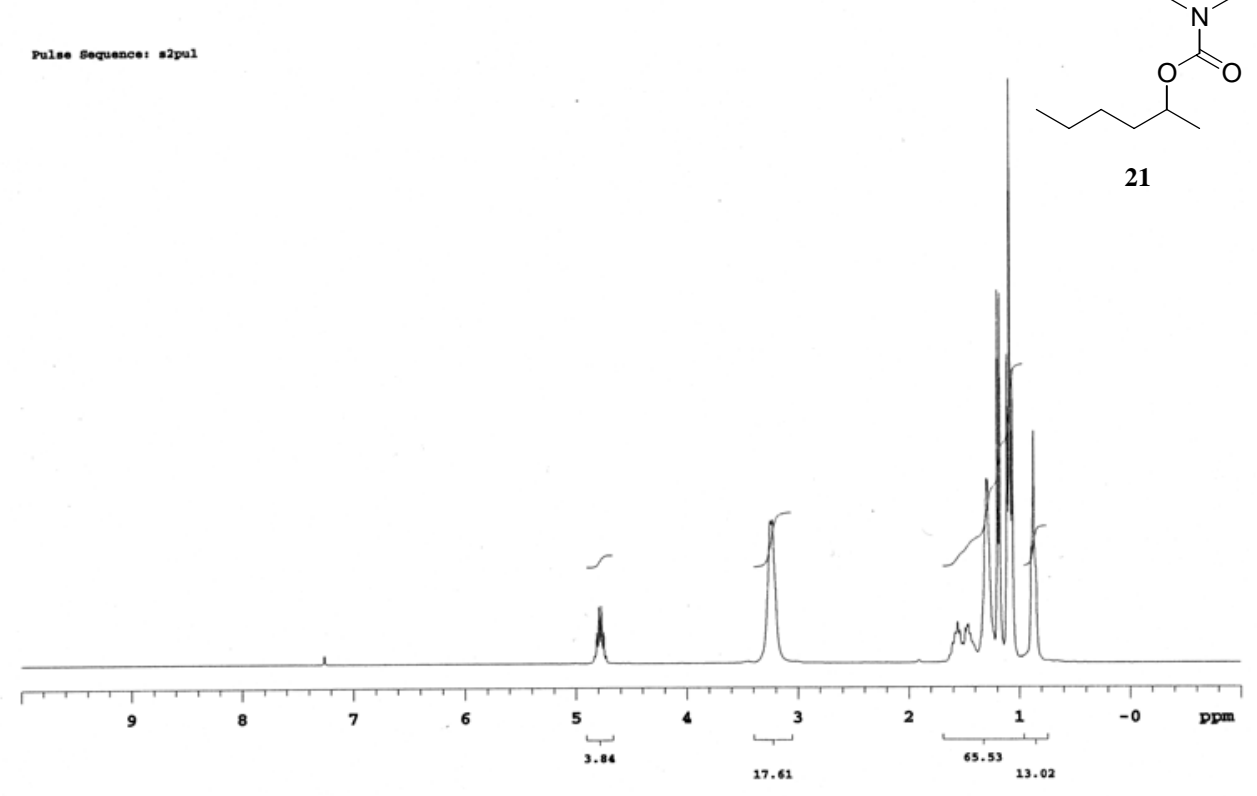


1-methyl-2-phenylethyl diethylcarbamate (22)
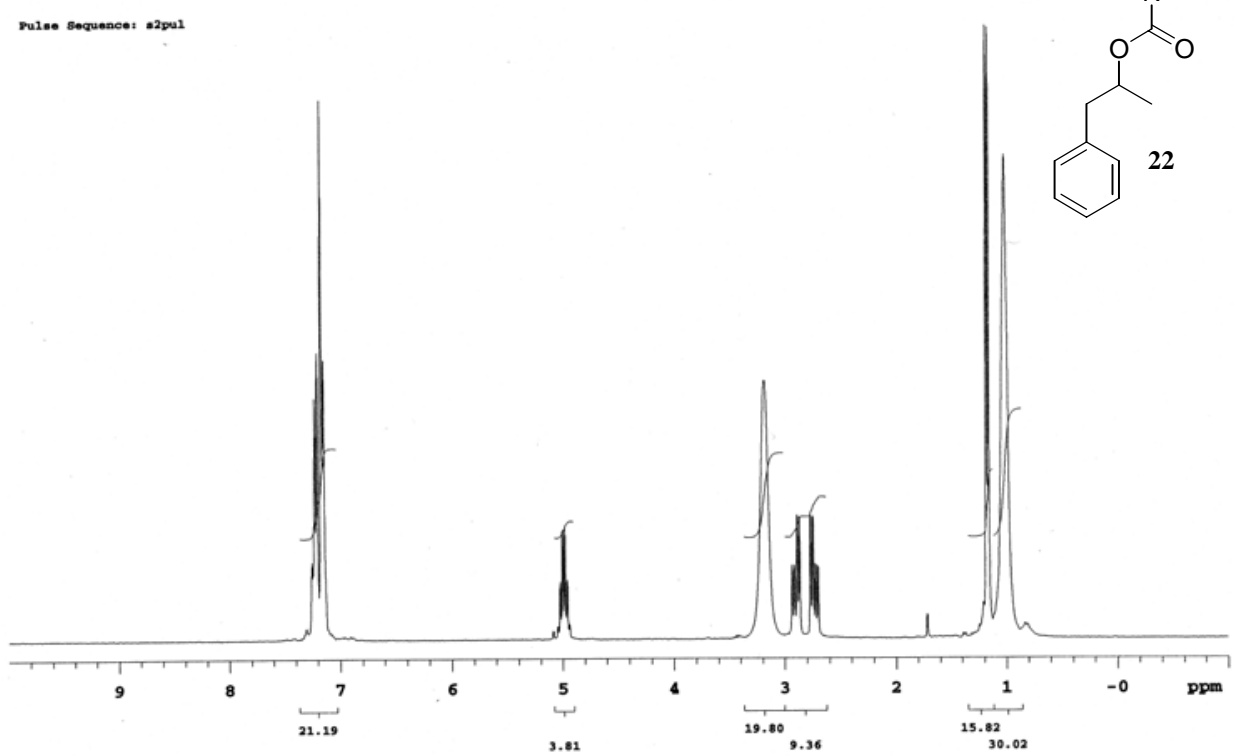

1-trimethylsilanylethyl diethylcarbamate (23)

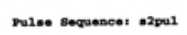

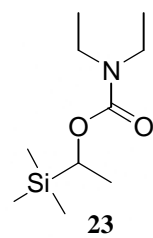

23

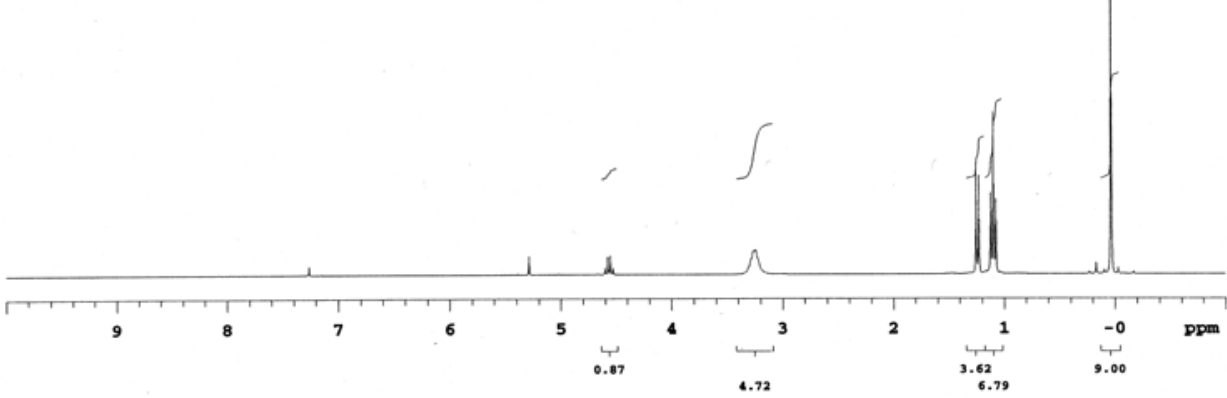


1-methyl-oct-2-enyl diethylcarbamate (24)

proles sequenoser, szpol
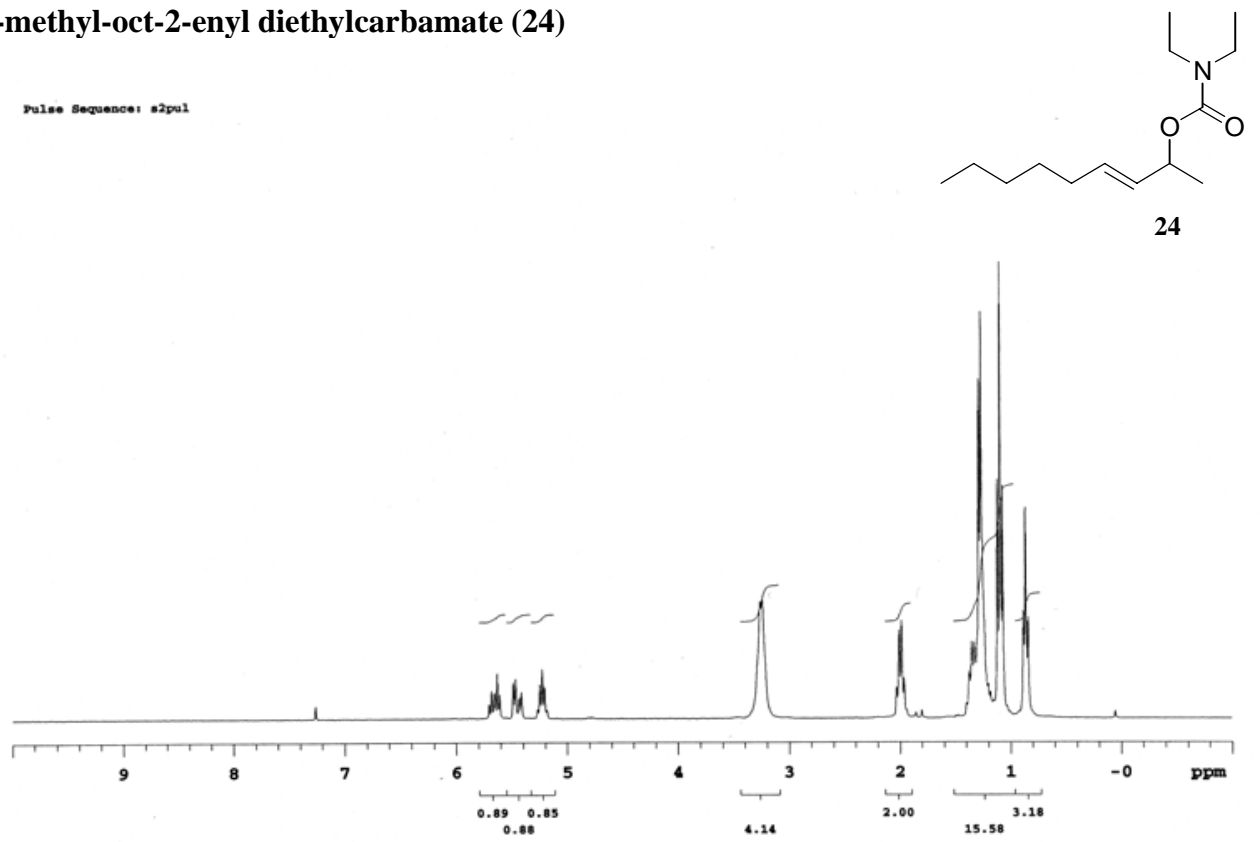

1-methyl-3-phenyl-allyl diethylcarbamate (25)

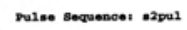
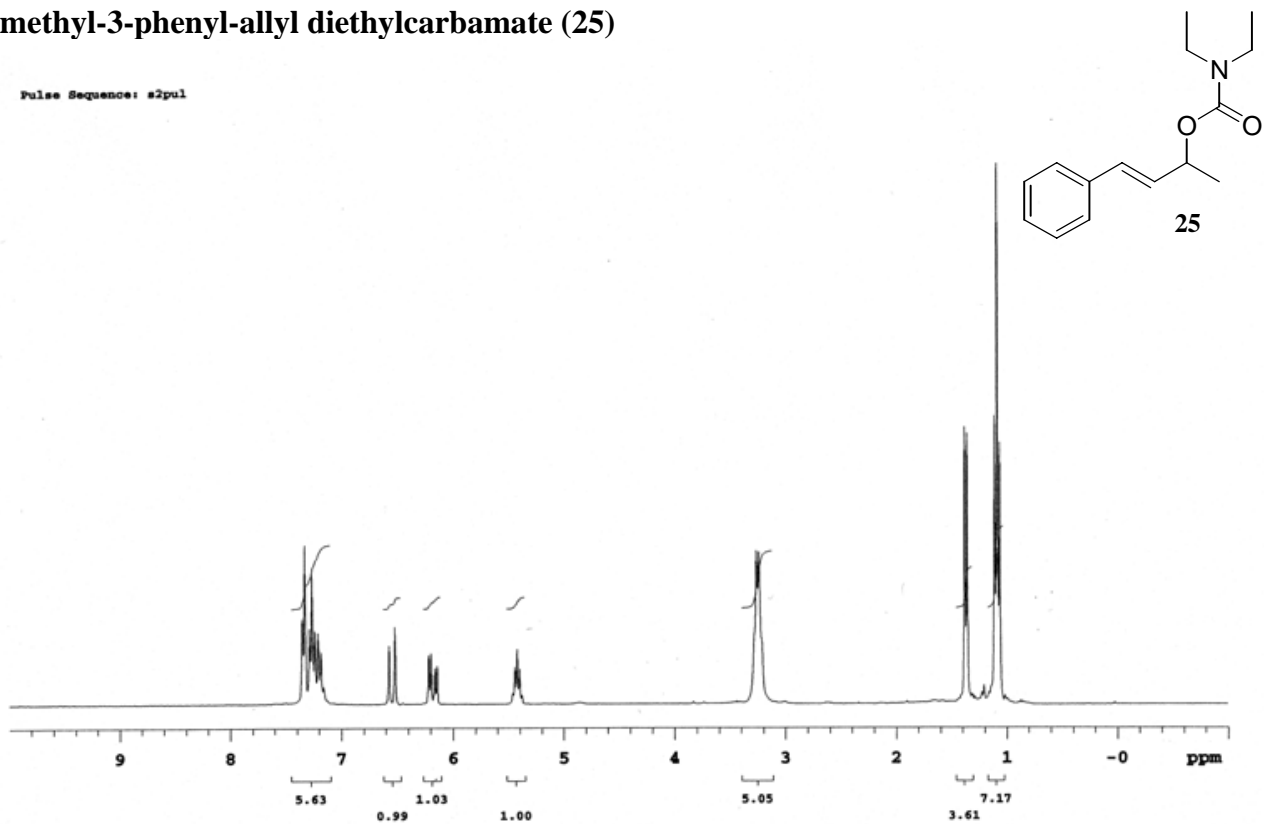
1-phenylvinyl dimethylcarbamate (7)

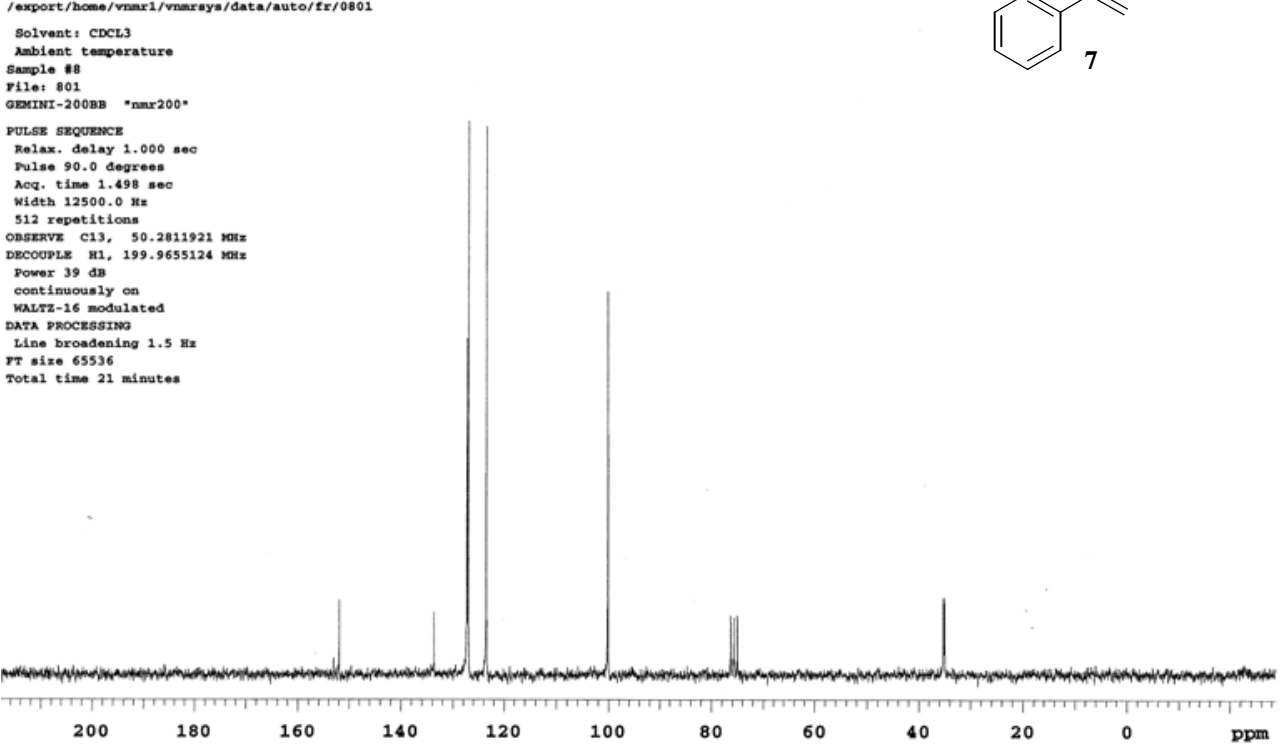

1-phenylvinyl diethylcarbamate (8)

/export/bceno/vmmr1/vanrays/data/auto/tw/0701

solvent! CDCL3

Mnbient tesperature

File: 701

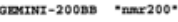

poLse azpuence

Relax. delay $1.000 \mathrm{sec}$

palse 90.0 degrees

widet $12500,0 \mathrm{~Hz}$

1600 repotition

OBszave e13, 50.2811921 me

DECOUPLE R1, 199.9655124 not:

continuous $1 \mathrm{y}$.

continuous ly on
KaLIZ-16 modulated

DRTA PROCESSTMO

Line broadening $1.5 \mathrm{~Hz}$

ry
rotal timo 66 minutes
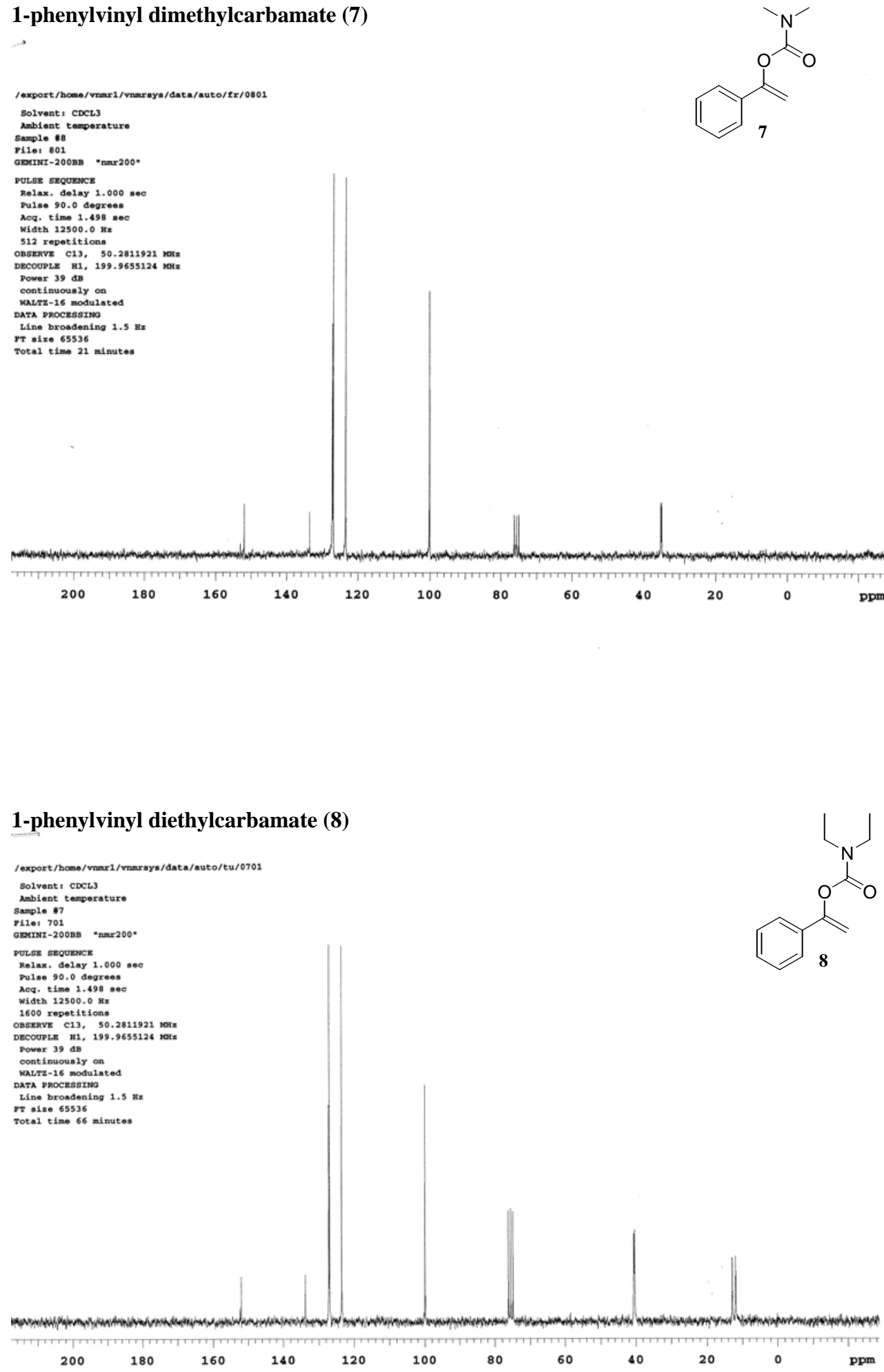
1-phenylvinyl diisopropylcarbamate (9)

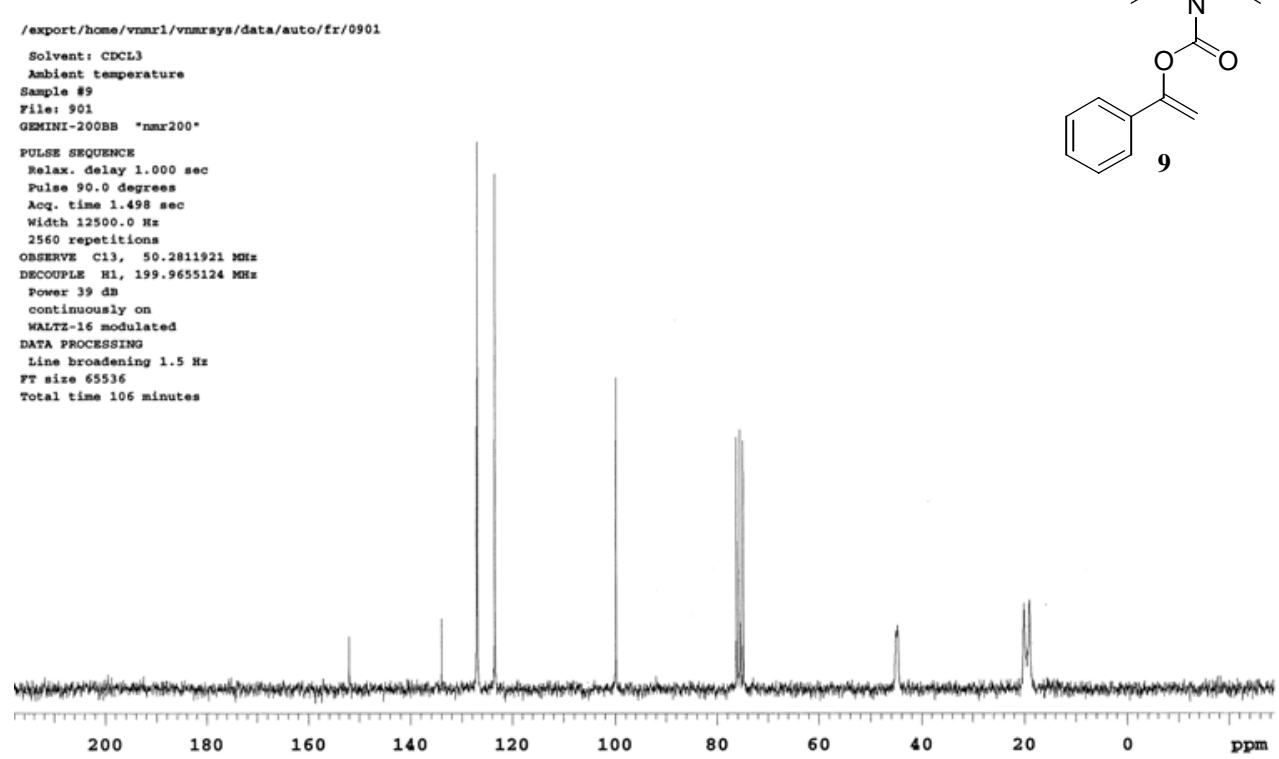

1-phenylethyl dimethylcarbamate (10)
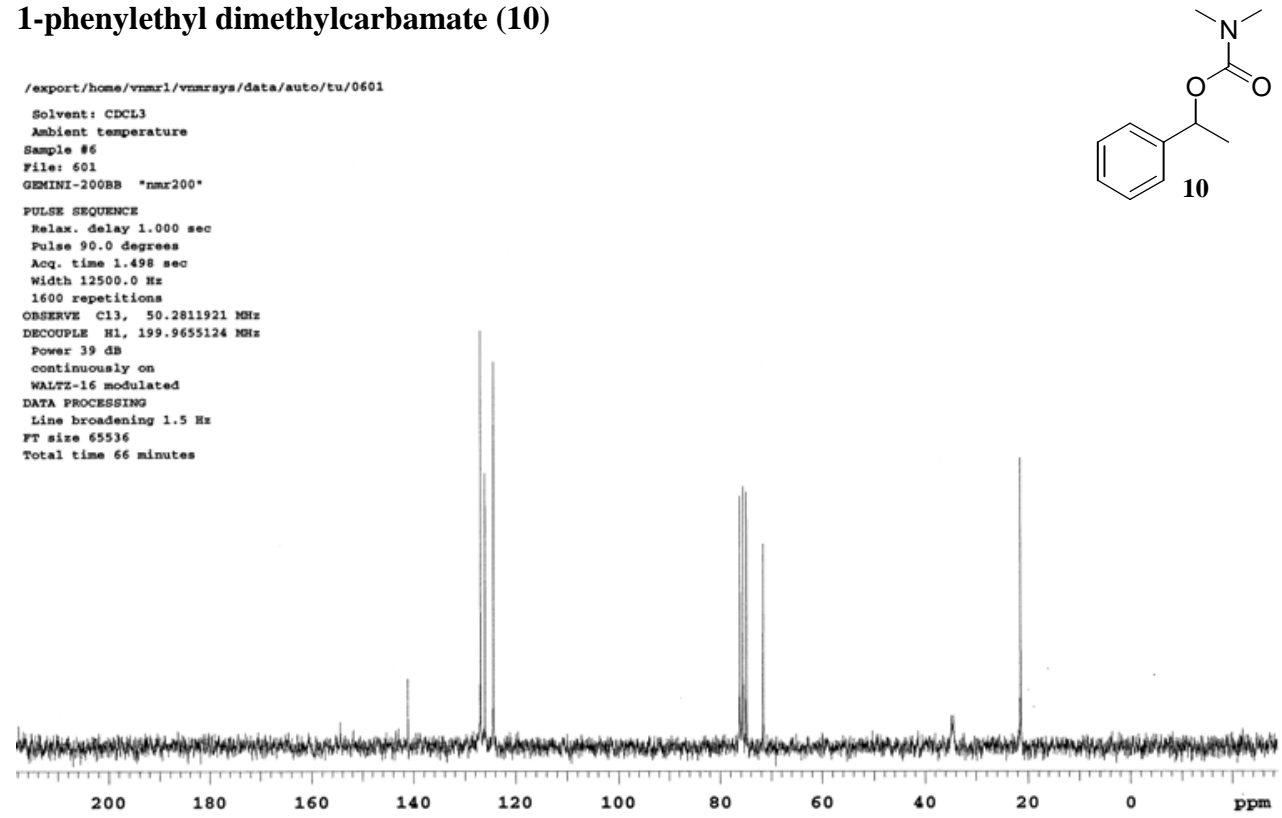
1-phenylethyl diethylcarbamate (11)

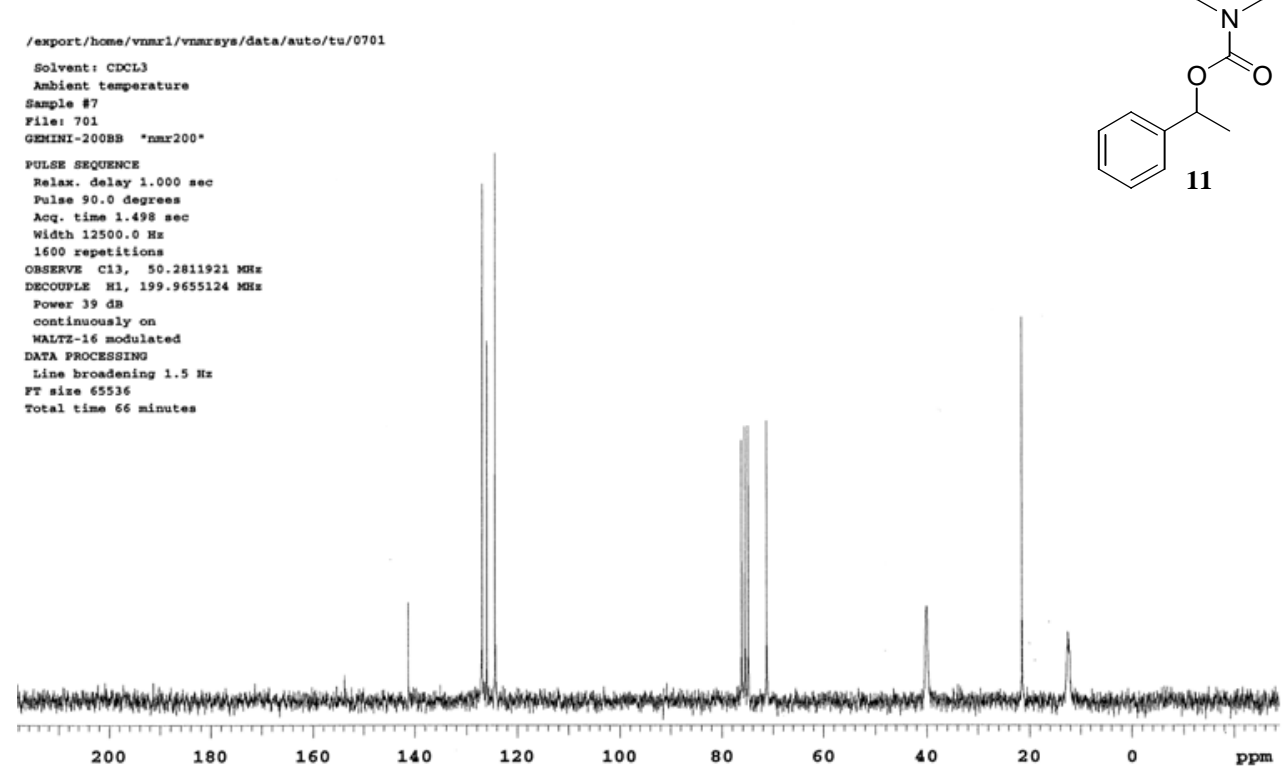

1-(4-nitro-phenyl)-vinyl diethylcarbamate (13)

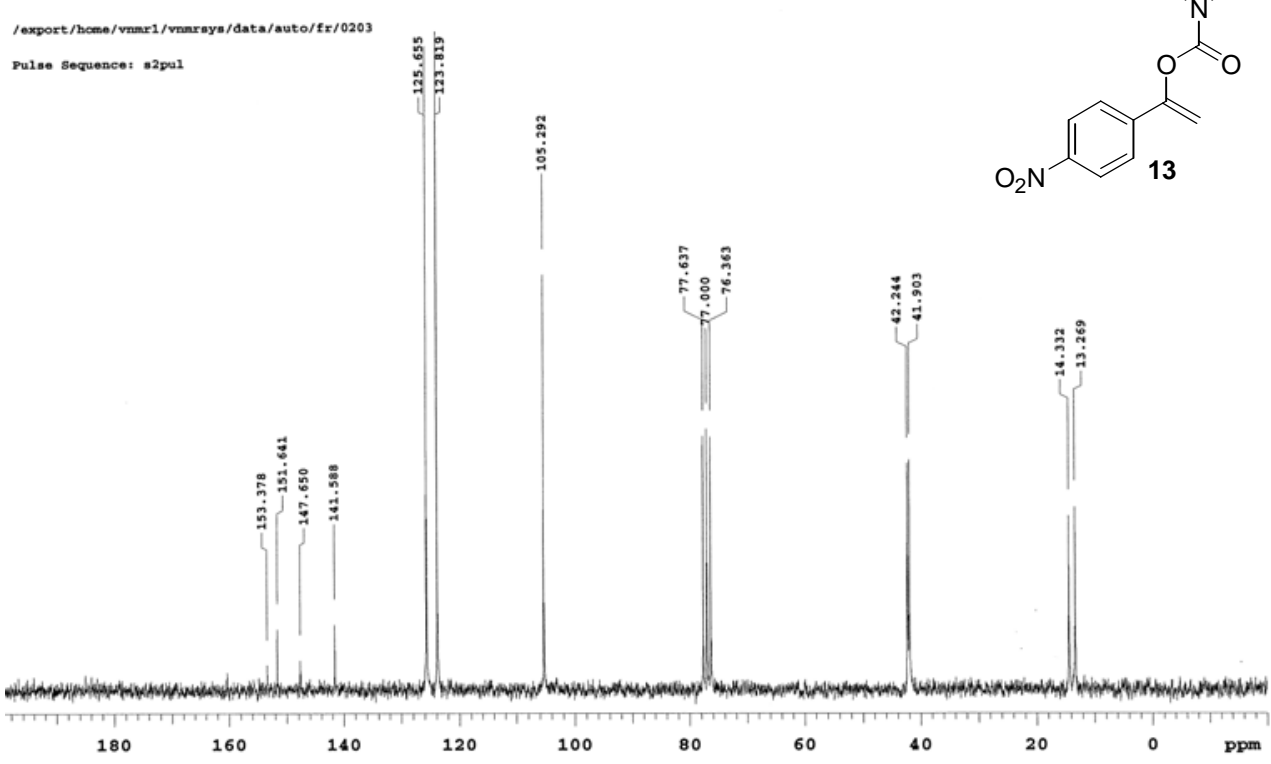


1-butylvinyl diethylcarbamate (14)

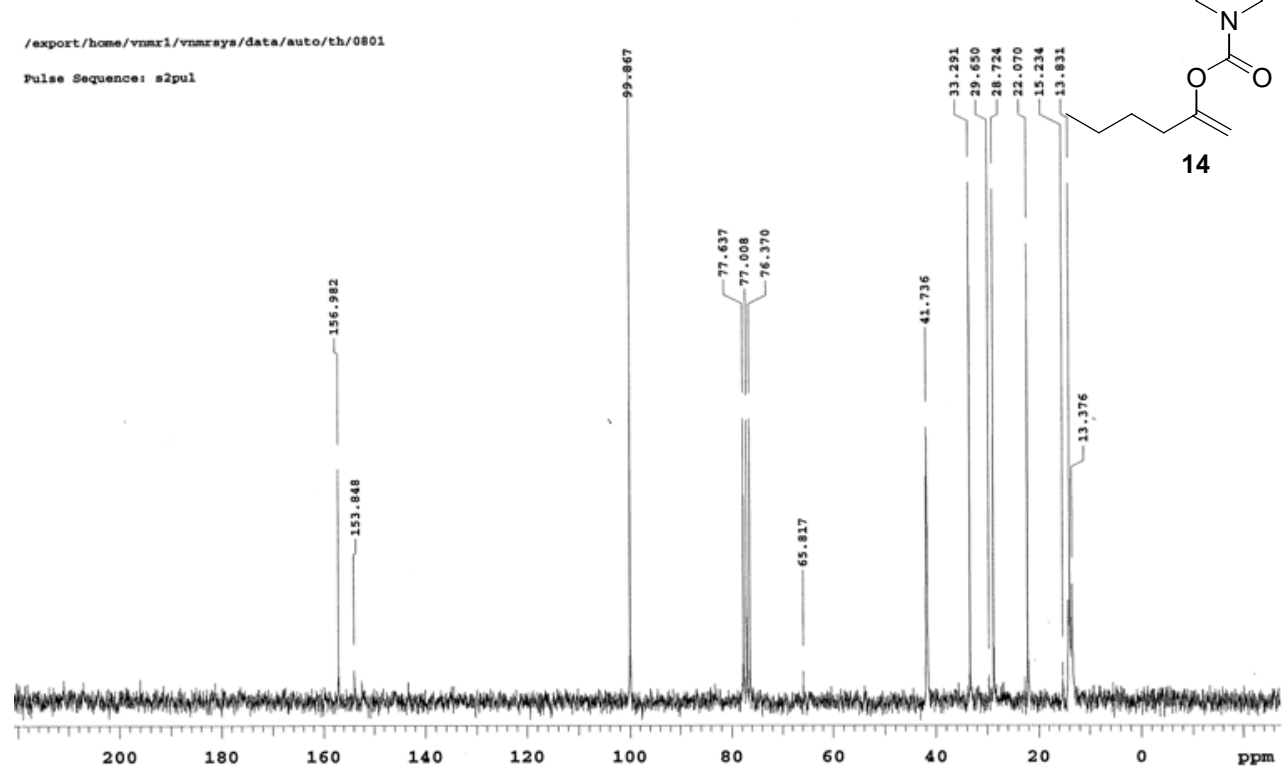

1-benzylvinyl diethylcarbamate (15)

/export/homo/vmmr1/vanrsys/data/auto/tr/0501

solvent! CDCL3

rambient compors

sample 55

Gratert-200BD "nmr200-

porse gequence

Pulsos 90.0 degrees

width $12500.0 \mathrm{~Hz}$

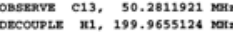

prover 39 dB

WALIZ-16 modulate

DATA PHOCRSsmo

Line brosdening $1.5 \mathrm{Rz}$

rT 8 ize 65536 (n)

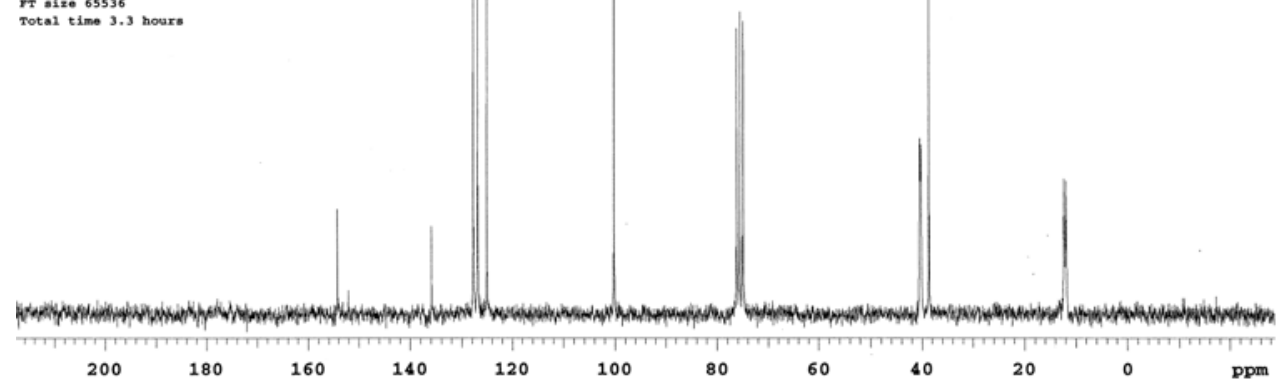


1-trimethylsilanylvinyl diethylcarbamate (16)

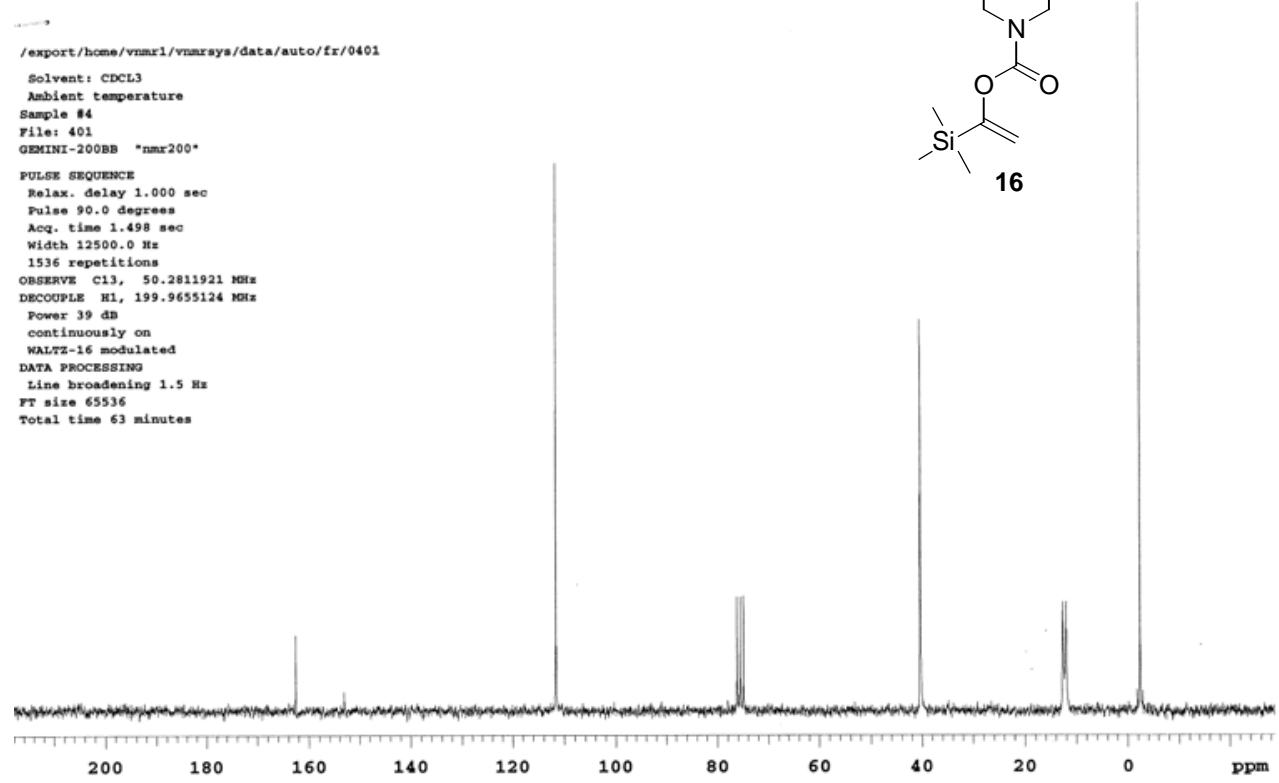

1-methylene-oct-2-enyl diethylcarbamate (17)

pulso sequencer sapul
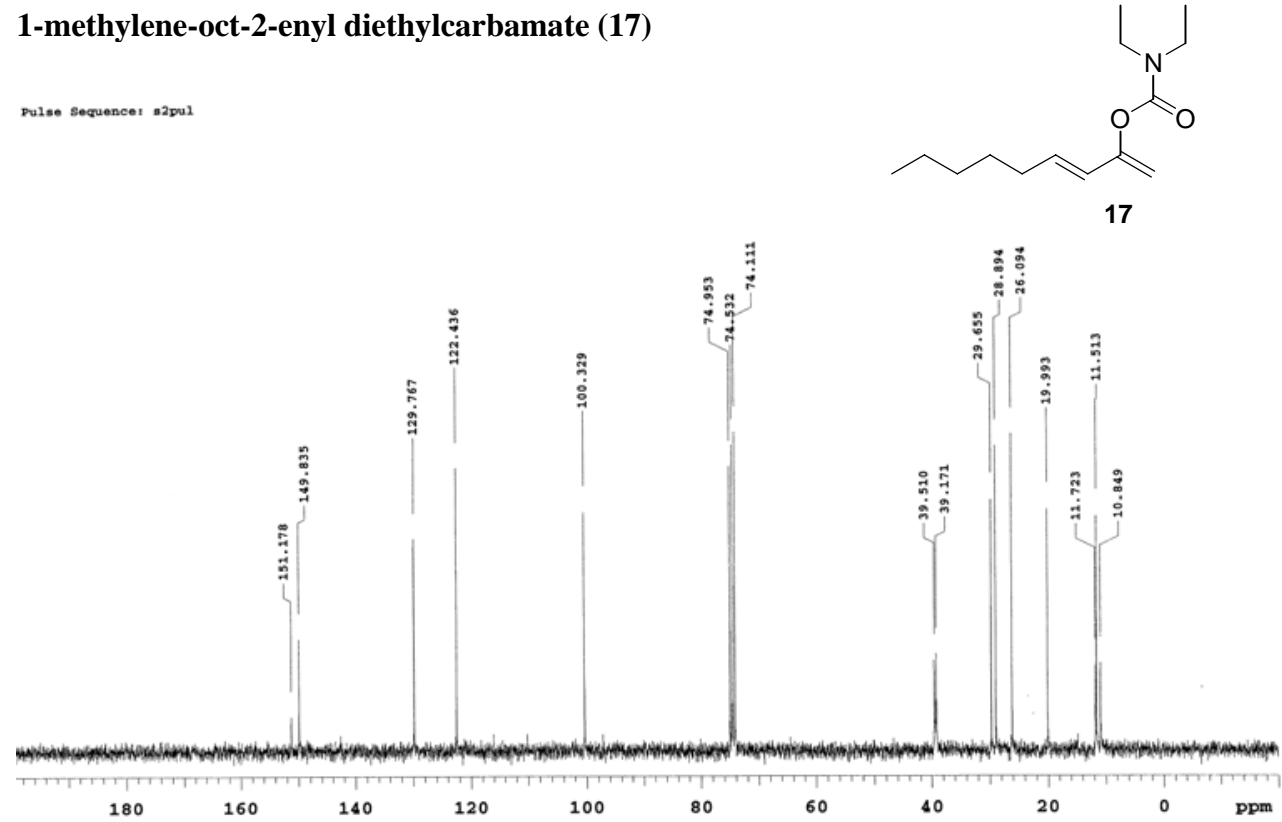
1-methylene-3-phenyl-allyl diethylcarbamate (18)

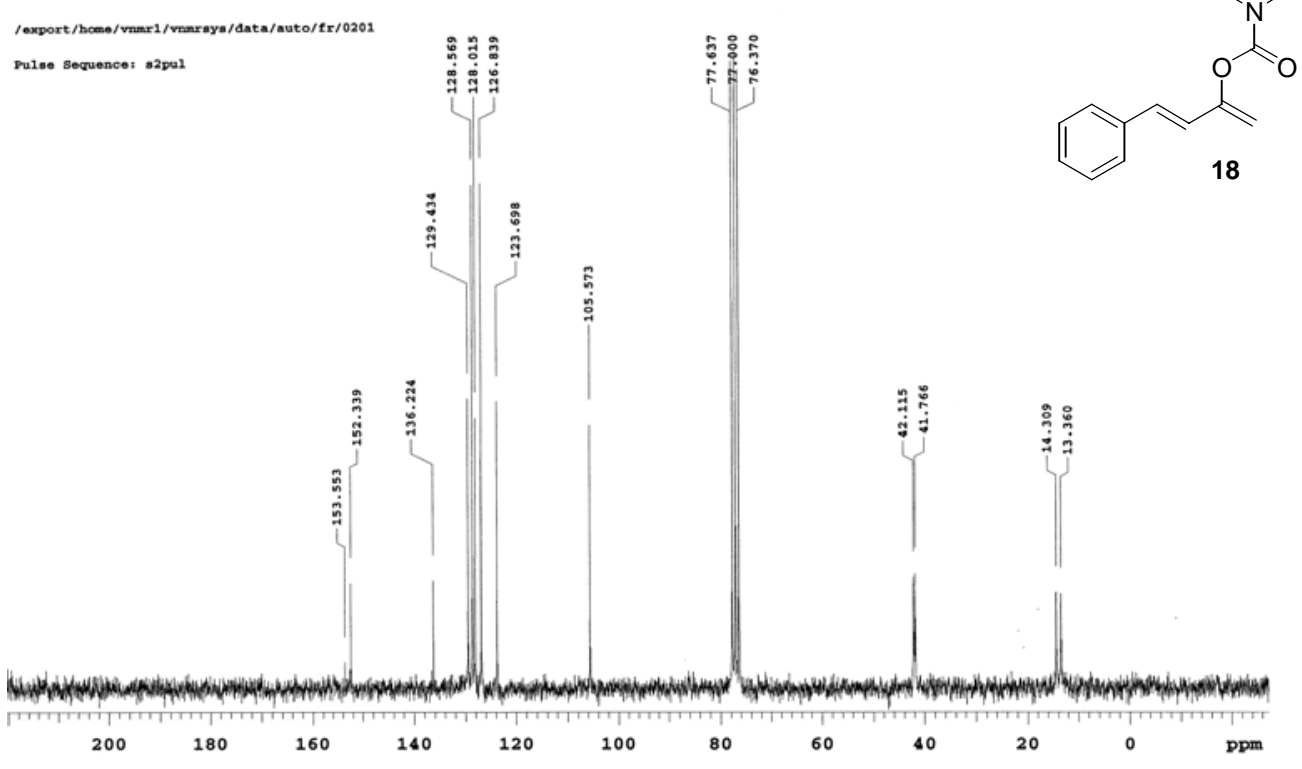

vinyl diethylcarbamate (19)

Pu1se sequence: s2pul

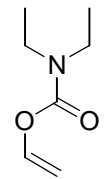

19

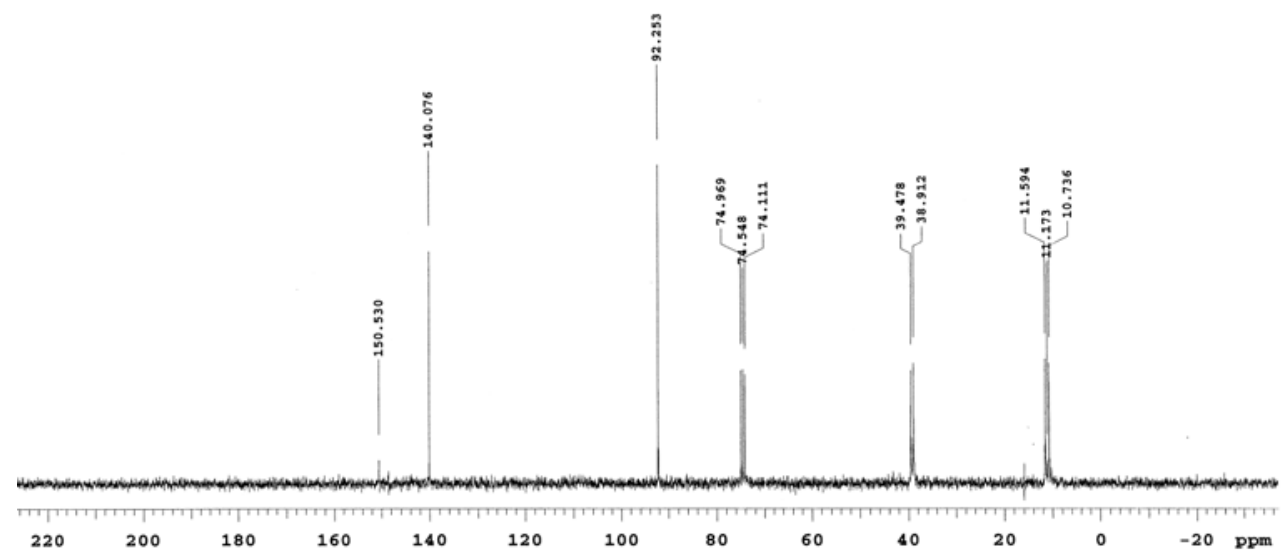




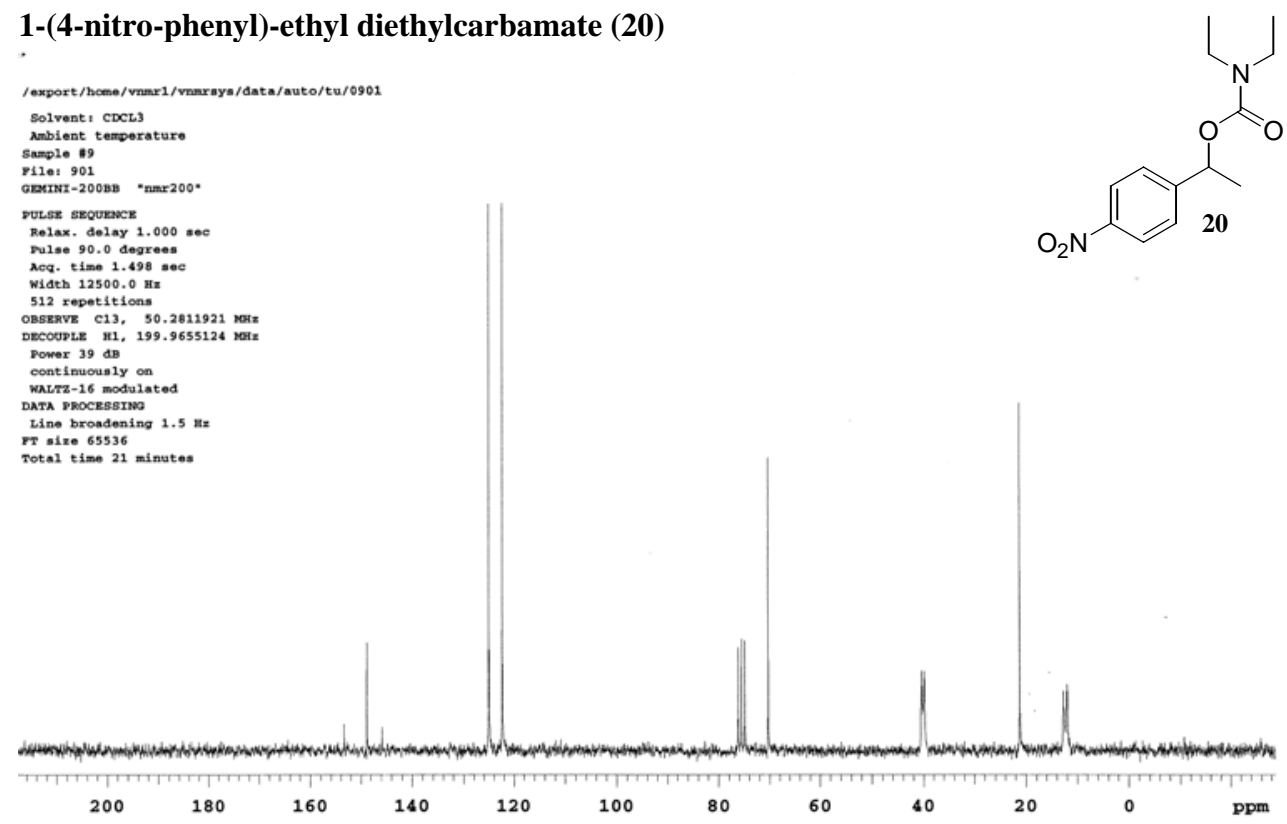

1-methylpentyl diethylcarbamate (21)

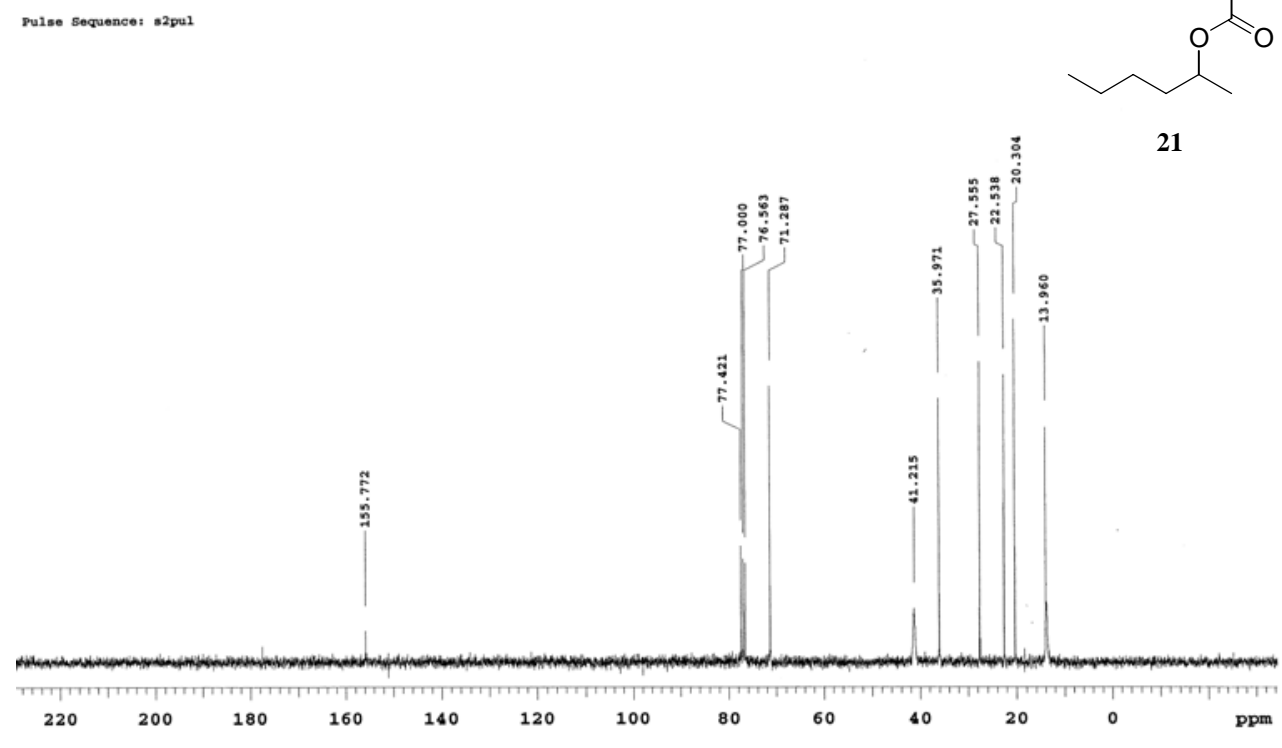


1-methyl-2-phenylethyl diethylcarbamate (22)

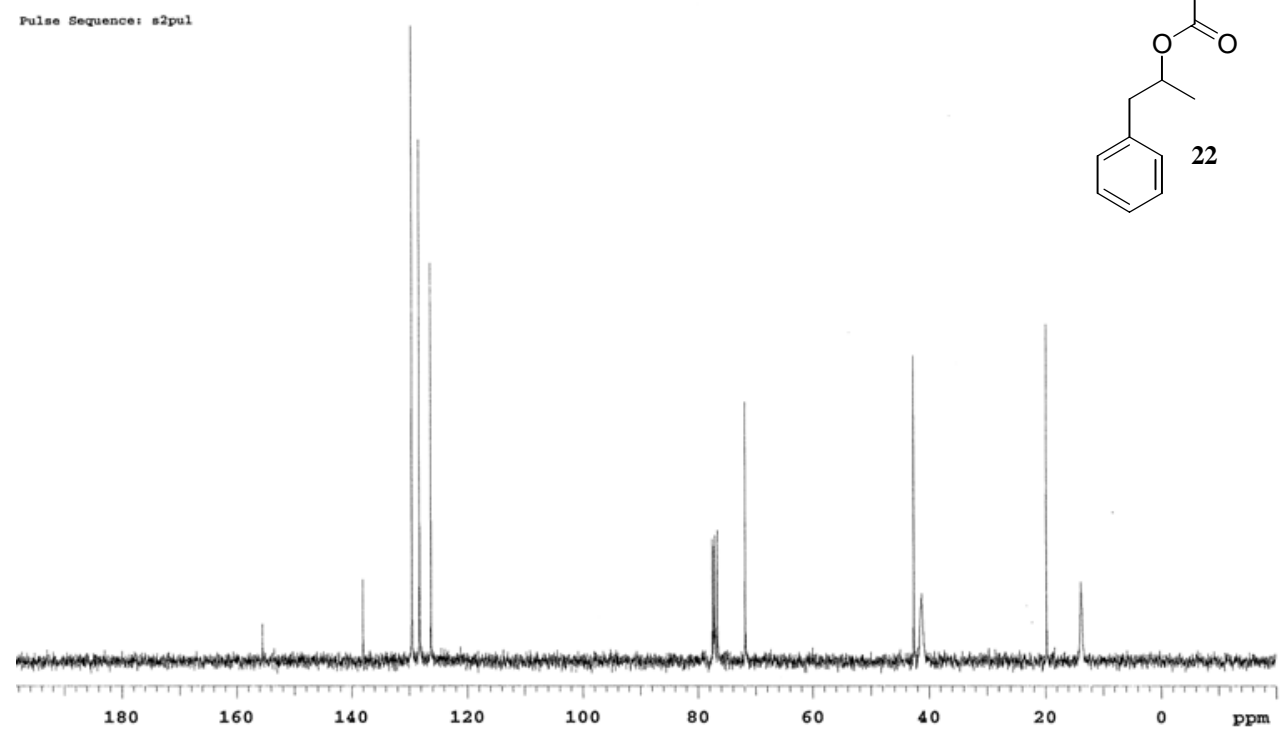

1-trimethylsilanylethyl diethylcarbamate (23)
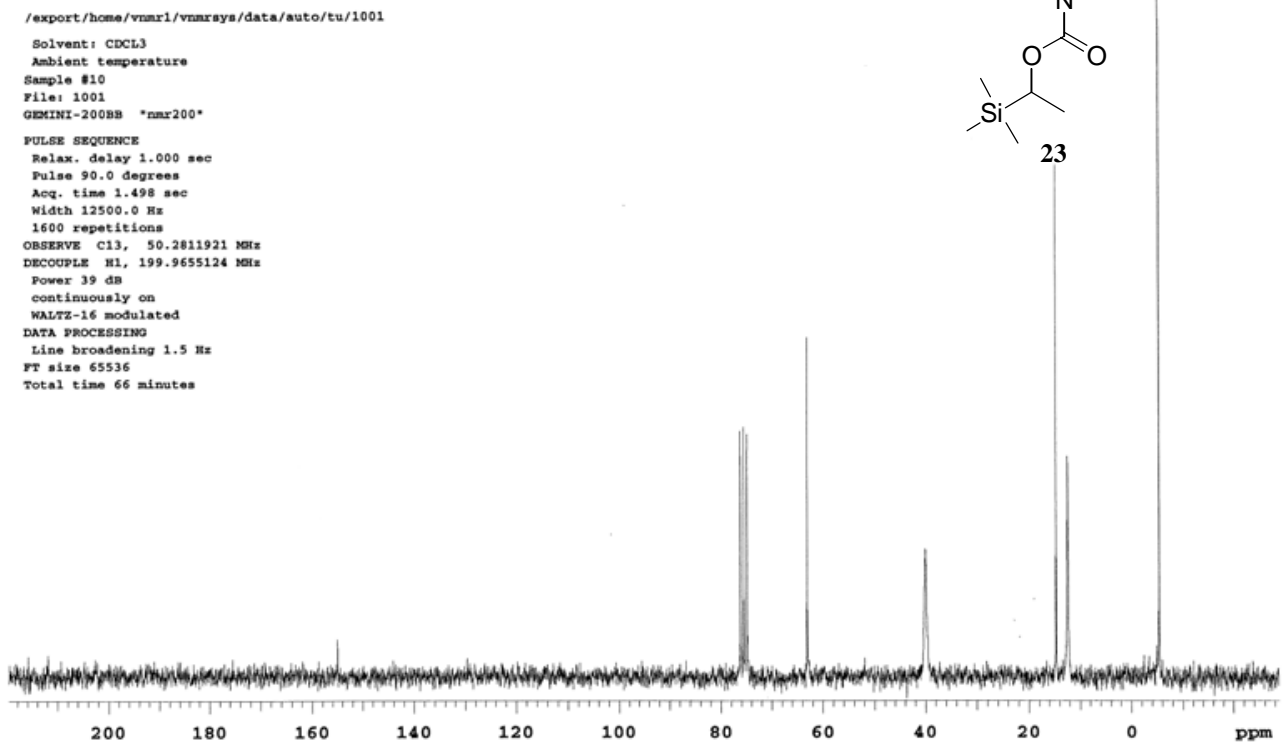
1-methyl-oct-2-enyl diethylcarbamate (24)

Pu1se Sequence: :20ul
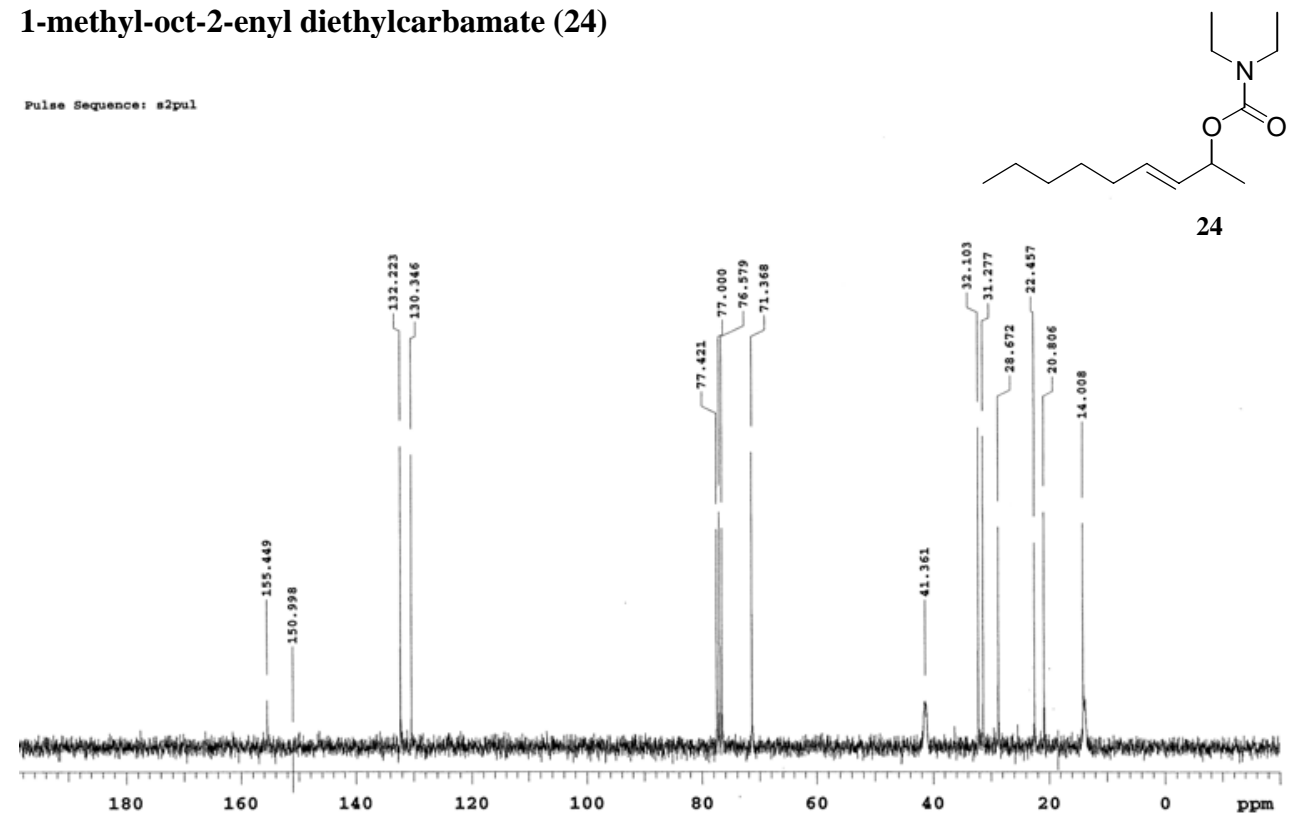

1-methyl-3-phenyl-allyl diethylcarbamate (25)

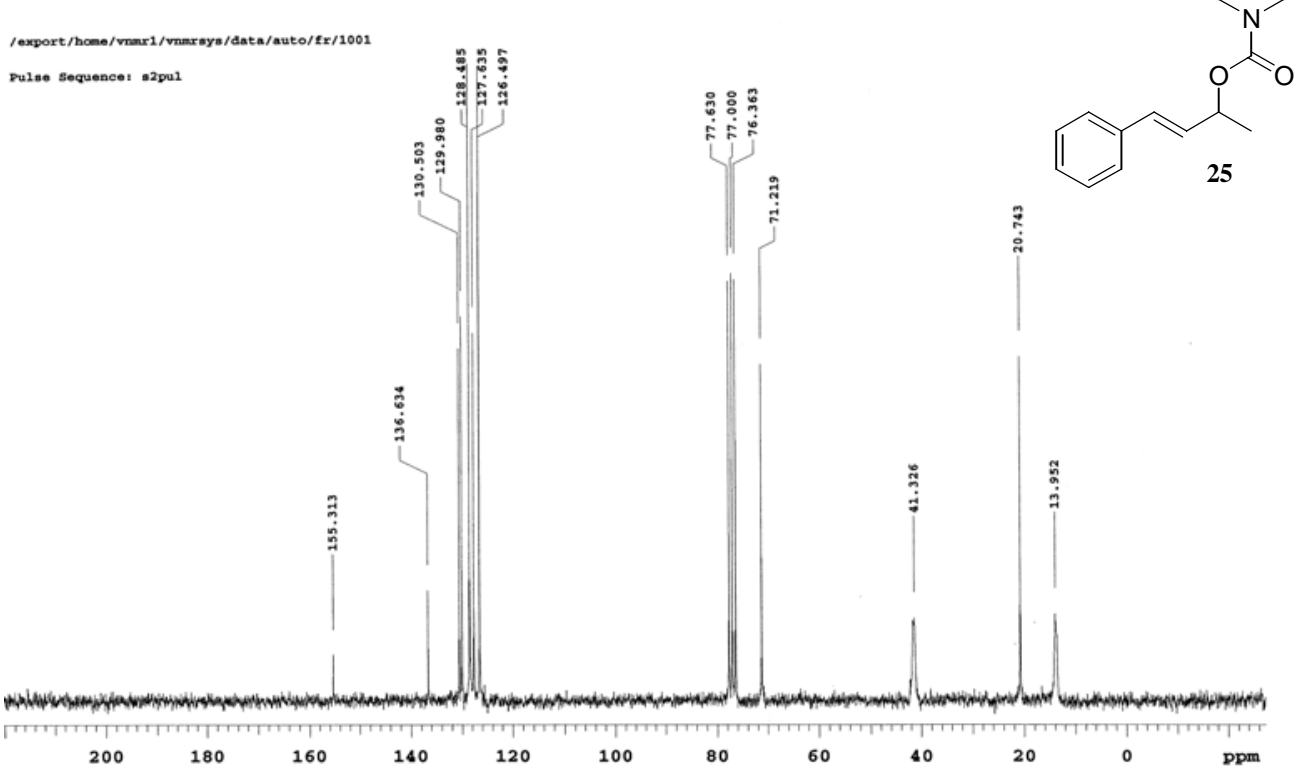

